\title{
DIVERSITY OF MOTHS (LEPIDOPTERA: HETEROCERA) IN THE SURROUNDINGS OF THE BEDNJA RIVER, VARAŽDIN COUNTY, NORTHERN CROATIA
}

\section{Toni Koren}

Hyla Association, I. Lipovac 7, HR-10000 Zagreb, Croatia (e-mail: toni.koren@hhdhyla.hr)

Koren, T.: Diversity of moths (Lepidoptera: Heterocera) in the surroundings of the Bednja River, Varaždin County, Northern Croatia. Nat. Croat., Vol. 27, No. 1, 111-141, Zagreb, 2018.

The first survey of the moth fauna on the Natura 2000 site Livade uz Bednju I to IV and its near surroundings was conducted in 2016. Four locations were selected and visited three times in different seasons. A total of 381 moth species were recorded, with the most numerous families being Geometridae (102 species), Noctuidae (94 species), Erebidae (44 species) and Crambidae (35 species). In order to put the results in perspective, a preliminary checklist of moths in the Varaždin county was created based on the literature overview and this survey, containing 603 species. 189 species recorded during this survey represent new county records. The greatest increase in the number of species was for the Noctuidae (40 species) and Geometridae (19 species) families. Some species like Diachrysia zosimi were previously recorded only a few times in Croatia. The surveyed sites can now be regarded as some of the best studied areas in Croatia in respect to their moth fauna.

Key words: wet meadows, Natura 2000, checklist, fauna

Koren, T.: Raznolikost noćnih leptira (Lepidoptera: Heterocera) u okolici rijeke Bednje, Varaždinska županija, sjeverna Hrvatska. Nat. Croat., Vol. 27, No. 1, 111-141, Zagreb, 2018.

Tijekom 2016. godine provedeno je prvo istraživanje noćnih leptira na Natura 2000 lokacijama Livade uz Bednju I - IV i u bližoj okolici. Četiri odabrane lokacije posjećene su po tri puta u različitim godišnjim dobima. Ukupno je zabilježeno 381 vrsta noćnih leptira, a najbrojnije porodice bile su Geometridae (120 vrsta), Noctuidae ( 94 vrste), Erebidae (44 vrste) te Crambidae (35 vrsta). Kako bismo stavili podatke u perspektivu, temeljem dostupne literature i ovog istraživanja izrađen je preliminarni popis noćnih leptira Varaždinske županije koji trenutno broji 603 vrste. Od vrsta zabilježenih ovim istraživanjem 189 ih je po prvi puta pronađeno na području županije. Najviše dodatnih vrsta zabilježeno je za porodice Noctuidae (40 vrsta) i Geometridae (19 vrsta). Neke vrste poput Diachrysia zosimi zabilježene su svega nekoliko puta na području Hrvatske. Istraživane se lokacije mogu smatrati nekim od najbolje istraženih lokacija u Hrvatskoj s obzirom na faunu noćnih leptira.

Ključne riječi: vlažne livade, Natura 2000, popis, fauna

\section{INTRODUCTION}

Moths are a numerous and highly diverse group of insects. They are important plant pollinators with more than two hundred different moth-plant interactions described so far (HAHN \& BRÜHL, 2016). Moths also represent prey for many invertebrate and vertebrate species (Kitching et al., 2000; Summerville \& Crist, 2004).

The moth fauna of most parts of Croatia has not been the subject of systematic faunistic studies in the past and the same is true for Varaždin County. Only a few references 
containing a very limited amount of, in almost all cases, more than a century-old data are available (e.g. JuRINAC, 1884; TABORSKY, 1910; GRUND, 1918). In the work of JuRINAC (1884) the general term "Varaždin" is used for the locality, with no precise locations mentioned, according to the common practice at that time. TABORSKY (1910) lists only some dozens of species that he recorded during his short visit to the area and GRUND (1918) mentions only a number of Microlepidoptera species from the area. Recent papers dealing with the moth fauna of the county were the results of the revision of Franjo Koščec's insect collection currently stored in the Entomological Department of Varaždin City Museum. The collection is extremely rich in all the insect groups, including butterflies and moths. The data from the Koščec butterfly collection were completely published (MARčEc, 2006), while only some of the moths from the Koščec collection have been published to date, including the Geometridae (Minoci, 2008), Noctuidae (Kučinić \& Bregovic, 1996) and the Sesiidae (KuČinić et al., 1997; PredovniK, 2010). Nevertheless, almost all the mentioned publications were based on specimens collected at the beginning of the $20^{\text {th }}$ century. Their historical value is unquestionable but they do not represent the current state of the biodiversity of the area. The goal of this survey was to gain a baseline insight into the moth diversity of the surroundings of the Bednja River, Varaždin County. Additionally, in order to put the collected data in perspective, the secondary goal was to create a preliminary checklist of moth species known from the county based on the available literature and this survey.

\section{MATERIALS AND METHODS}

\section{Study site}

Varaždin County has an area of $1262 \mathrm{~km}^{2}$ and 175951 inhabitants. The county contains only a few cities (Varaždin, Ivanec, Ludbreg, Lepoglava, Novi Marof and Varaždinske Toplice) and a large number of villages and small settlements. The whole area is a mosaic of settlements, agricultural land, forest fragments and grasslands of different kinds. The two largest rivers in the area are the Drava, which flows along the northern border of the county, and the Bednja which flows into the Drava within the county. The Bednja is a tributary of the Drava, and the whole of its course is in Croatia. The river is $133 \mathrm{~km}$ long and rises at Mt Macelj and flows through Bednja, Beletinec, Novi Marof, Varaždinske toplice, Ludbreg and joins the Drava at Mali Bukovac near Ludbreg.

A total of 14 Special Protection Areas (SPAs) sites have been designated in the county, including the Natura 2000 areas Livade uz Bednju I-V (site codes: HR2001408, HR2001409, HR2001410, HR2001411, and HR2001412) occupying a total area of 1,534.761 ha. The Bednja River and its valley constitute an important area of the ecological network, particularly because of the meadow and pasture habitats, and the associated species (ANONYMous, 2015). In the river's surroundings, several important habitats are present: lowland meadows (with Alopecurus pratensis and Sanguisorba officinalis), Molinia meadows on calcareous, peaty or clayey-silt-laden soils (Molinion caeruleae) and hydrophilic edges higher green with rivers and forests (Convolvulion sepia, Filipendulion, Senecion fluviatilis). The dominant soils are acidic brown soil on clastic rocks, luviziol on clay and partly hydroameliorated wetland rendzinas on flysch and soft limestone (ANONYMous, 2015). The Bednja River area and the surrounding habitat is one of last remaining natural sites in the county and, consequently large parts of the remaining mesophilous grasslands are included in the Natura 2000 network. Still, except for basic knowledge about the Natura 2000 species and habitats, almost no other available data exist for the area. 


\section{Moth survey}

This study took place at four localities in the Natura 2000 areas, Livade uz Bednju I - IV, or in the near surroundings (Fig. 1). The area Livade uz Bednju V was not included as it was not easily accessible by car. Each locality was visited three times in different seasons (spring, summer and autumn). On average, four hours were spent at each locality on each date, depending on the time of year and the climate conditions. Five to seven UV light tents were used per locality to attract moths. Most of the moths attracted were identified in the field and released. Only in cases where the identification was questionable were the moths collected and identified in the lab using standard identification keys (e.g. Fibiger, 1997; Hausmann \& Viddalepr, 2012; MaceK et al., 2012; Nowacki, 1998; Slamka, 2006, 2008, 2010, 2013). Some members of the families Erebiidae, Geometridae and Noctuidae, and all the encountered Microlepidoptera were collected and stored in the private collection of the author. The nomenclature follows the online database Fauna Europaea (DE JoNG et al., 2014). For several species, examination of the genital structures was carried out in order to confirm the correct species identification.

In order to validate the results and compare them with historical records, a preliminary checklist of moths of the county was created based on the available literature (Jurinac, 1884; TAborsky, 1910; Grund, 1918, Kučinić \& Bregović, 1996, Kučinić et al., 1997; Minoci, 2008; Predovnik, 2010).

Tab. 1. List of surveyed localities along with habitats, coordinates, altitude and dates of visits.

\begin{tabular}{|l|l|l|c|c|c|c|}
\hline \multicolumn{1}{|c|}{ Locality } & \multicolumn{1}{c|}{ Habitat } & X & Y & $\begin{array}{c}\text { Altitude } \\
\text { (m a.s.l.) }\end{array}$ & Dates of visit \\
\hline 1. & $\begin{array}{l}\text { Trakošćan, south of } \\
\text { Cvetlin Village }\end{array}$ & $\begin{array}{l}\text { forest edge, wet } \\
\text { meadows }\end{array}$ & 46,268605 & 15,950217 & 252 & $\begin{array}{c}9.5 .2016,12.7 .2016, \\
5.10 .2016\end{array}$ \\
\hline 2. & Ivanec, lakes & $\begin{array}{l}\text { forest edge, wet } \\
\text { meadows, edge of } \\
\text { the lakes }\end{array}$ & 46,230913 & 16,102167 & 207 & $\begin{array}{c}6.4 .2016,11.7 .2016, \\
14.11 .2016\end{array}$ \\
\hline 3. & $\begin{array}{l}\text { Novi Marof, Ivci, } \\
\text { riverbank of Bednja }\end{array}$ & $\begin{array}{l}\text { forest edge, wet } \\
\text { meadows }\end{array}$ & 46,153744 & 16,364668 & 187 & $\begin{array}{c}7.6 .2016,18.7 .2016, \\
23.10 .2016\end{array}$ \\
\hline 4. & $\begin{array}{l}\text { Donji Martijanec, } \\
\text { east of the village }\end{array}$ & $\begin{array}{l}\text { forest edge, wet } \\
\text { meadows }\end{array}$ & 46,230737 & 16,552234 & 168 & $\begin{array}{c}18.5 .2016,25.8 .2016, \\
2.11 .2016\end{array}$ \\
\hline
\end{tabular}

\section{RESULTS}

During this survey we gathered data of 381 moth species within the study area. The Geometridae are the best-represented group with a total of 102 recorded species, followed by Noctuidae with 94 species, Erebiidae with 44 and Crambidae with 35 species. All four localities had a similar number of species and abundance of specimens across three different seasons (Fig. 2).

From the available literature a list of 414 moth species from the Varaždin County was created. Overall, 189 species recorded during this survey represent new county records andwhen they are included the known number of moths occurring in Varaždin County is actually 603 (Appendix I). 


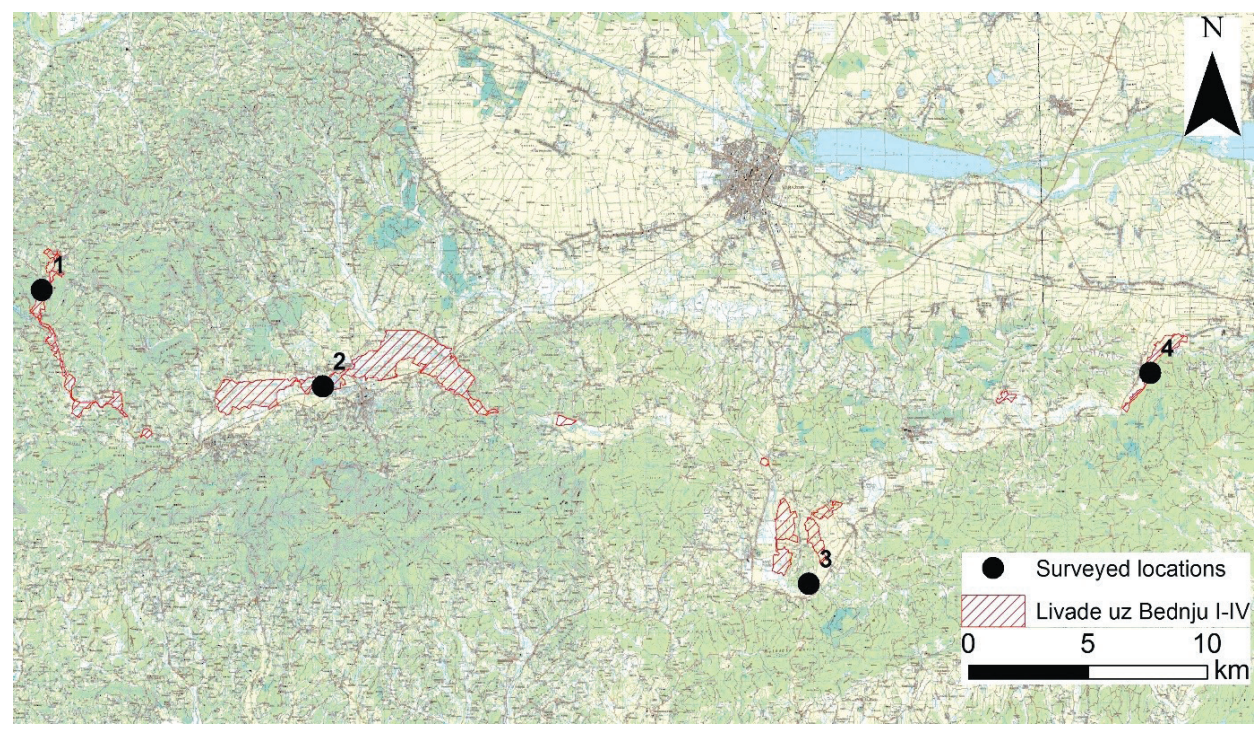

Fig. 1. Surveyed locations in the Natura 2000 areas Livade uz Bednju I-IV. Locality numbers follow Tab. 1.

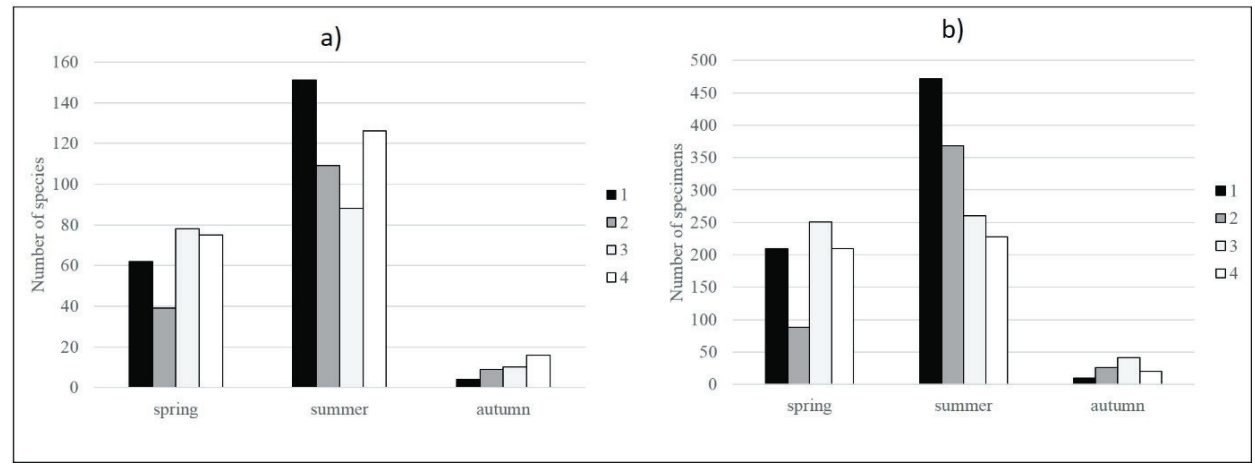

Fig. 2. Number of species (a) and specimens (b) per each locality and season. Locality numbers follow Tab. 1 .

Tab. 2. List of recorded moth species during this survey. The locality numbers correspond to the ones given in Tab. 1. Species for which the identification is based on the examination of the genital structures are marked with an * symbol.

\begin{tabular}{|c|c|c|c|c|c|c|}
\hline \multirow{2}{*}{\multicolumn{2}{|c|}{ Species }} & \multicolumn{4}{|c|}{ Location number } & \multirow{2}{*}{$\begin{array}{l}\text { Total No. of } \\
\text { individuals }\end{array}$} \\
\hline & & 1 & 2 & 3 & 4 & \\
\hline & \multicolumn{6}{|l|}{ Chimabachidae } \\
\hline 1. & Diurnea fagella ([Denis \& Schiffermüller], 1775) & & 6 & & & 6 \\
\hline \multirow[t]{2}{*}{2.} & Diurnea lipsiella ([Denis \& Schiffermüller], 1775) & 1 & & & & 1 \\
\hline & \multicolumn{6}{|l|}{ Cossidae } \\
\hline 3. & Cossus cossus (Linnaeus, 1758) & 1 & & 5 & & 6 \\
\hline \multirow[t]{2}{*}{4.} & Zeuzera pyrina (Linnaeus, 1761) & & 3 & & & 3 \\
\hline & Crambidae & & & & & \\
\hline 5. & Agrotera nemoralis (Scopoli, 1763) & 3 & 1 & & 1 & 5 \\
\hline
\end{tabular}




\begin{tabular}{|c|c|c|c|c|c|c|}
\hline \multirow{2}{*}{\multicolumn{2}{|c|}{ Species }} & \multicolumn{4}{|c|}{ Location number } & \multirow{3}{*}{$\begin{array}{l}\text { Total No. of } \\
\text { individuals } \\
3\end{array}$} \\
\hline & & \multirow{2}{*}{1} & \multirow{2}{*}{2} & \multirow{2}{*}{3} & \multirow{2}{*}{$\begin{array}{l}4 \\
3\end{array}$} & \\
\hline 6. & Anania coronata (Hufnagel, 1767) & & & & & \\
\hline 7. & Anania fuscalis ([Denis \& Schiffermüller], 1775) & & & 5 & & 5 \\
\hline 8. & Anania lancealis ([Denis \& Schiffermüller], 1775) & & & & 2 & 2 \\
\hline 9. & Anania stachydalis (Germar, 1821) & 2 & 1 & 1 & 1 & 5 \\
\hline 10. & Anania verbascalis ([Denis \& Schiffermüller], 1775) & 5 & & 1 & 2 & 8 \\
\hline 11. & Calamotropha paludella (Hübner, 1824)* & & 5 & & & 5 \\
\hline 12. & Cataclysta lemnata (Linnaeus, 1758) & & 5 & 5 & 1 & 11 \\
\hline 13. & Catoptria osthelderi (Lattin, 1950)* & 5 & & & & 5 \\
\hline 14. & Chrysoteuchia culmella (Linnaeus, 1758) & 3 & 1 & & & 4 \\
\hline 15. & Crambus lathoniellus (Zincken, 1817) & & & 6 & & 6 \\
\hline 16. & Cynaeda dentalis ([Denis \& Schiffermüller], 1775) & & 1 & & & 1 \\
\hline 17. & Diasemia reticularis (Linnaeus, 1761) & & & & 1 & 1 \\
\hline 18. & Ecpyrrhorrhoe rubiginalis (Hübner, 1796) & & & & 5 & 5 \\
\hline 19. & Evergestis forficalis (Linnaeus, 1758) & 5 & & & 1 & 6 \\
\hline 20. & Evergestis pallidata (Hufnagel, 1767) & & & & 3 & 3 \\
\hline 21. & Metasia ophialis (Treitschke, 1829) & & & 2 & & 2 \\
\hline 22. & Myelois circumvoluta (Fourcroy, 1785) & & & 3 & & 3 \\
\hline 23. & Nomophila noctuella ([Denis \& Schiffermüller], 1775) & & & 1 & 1 & 2 \\
\hline 24. & Nymphula nitidulata (Hufnagel, 1767) & & & & 1 & 1 \\
\hline 25. & Ostrinia nubilalis (Hübner, 1796) & 2 & 3 & 5 & 1 & 11 \\
\hline 26. & Palpita vitrealis (Rossi, 1794) & & & & 2 & 2 \\
\hline 27. & Paracorsia repandalis ([Denis \& Schiffermüller], 1775) & & 3 & & & 3 \\
\hline 28. & Parapoynx stratiotata (Linnaeus, 1758) & & & 3 & & 3 \\
\hline 29. & Paratalanta pandalis (Hübner, 1825) & 5 & & & & 5 \\
\hline 30. & Pediasia contaminella (Hübner, 1796) & & & 5 & 1 & 6 \\
\hline 31. & Pleuroptya ruralis (Scopoli, 1763) & 5 & 4 & 5 & 2 & 16 \\
\hline 32. & Pyrausta aurata (Scopoli, 1763) & 5 & & & 1 & 6 \\
\hline 33. & Pyrausta despicata (Scopoli, 1763) & & & 2 & & 2 \\
\hline 34. & Pyrausta purpuralis (Linnaeus, 1758) & & & & 1 & 1 \\
\hline 35. & Sclerocona acutella (Eversmann, 1842) & 1 & 2 & & & 3 \\
\hline 36. & Scoparia pyralella ([Denis \& Schiffermüller], 1775) & & & 5 & & 5 \\
\hline 37. & Sitochroa verticalis (Linnaeus, 1758) & 1 & & & 1 & 2 \\
\hline 38. & Udea accolalis (Zeller, 1867) & 1 & & & & 1 \\
\hline \multirow[t]{2}{*}{39.} & Udea ferrugalis (Hübner, 1796) & 4 & 3 & 5 & 2 & 14 \\
\hline & \multicolumn{6}{|l|}{ Drepanidae } \\
\hline 40. & Drepana curvatula (Borkhausen, 1790) & 2 & & & 2 & 4 \\
\hline 41. & Drepana falcataria (Linnaeus, 1758) & 8 & 1 & & 3 & 12 \\
\hline 42. & Falcaria lacertinaria (Linnaeus, 1758) & 3 & 1 & & 1 & 5 \\
\hline 43. & Habrosyne pyritoides (Hufnagel, 1766) & 9 & & 5 & 2 & 16 \\
\hline 44. & Polyploca ridens (Fabricius, 1787) & & 1 & & & 1 \\
\hline 45. & Sabra harpagula (Esper, 1786) & 3 & & & & 3 \\
\hline 46. & Tethea or ([Denis \& Schiffermüller], 1775) & & & 2 & 1 & 3 \\
\hline 47. & Thyatira batis (Linnaeus, 1758) & 7 & 5 & & 1 & 13 \\
\hline 48. & Watsonalla binaria (Hufnagel, 1767) & & & 4 & & 4 \\
\hline \multirow[t]{2}{*}{49.} & Watsonalla cultraria (Fabricius, 1775) & & & & 1 & 1 \\
\hline & Elachistidae & & & & & \\
\hline 50. & Agonopterix ocellana (Fabricius, 1775) & & 20 & & & 20 \\
\hline
\end{tabular}


116 Koren, T.: Diversity of moths (Lepidoptera: Heterocera) in the surroundings of the Bednja river, Varaždin County

\begin{tabular}{|c|c|c|c|c|c|c|}
\hline \multirow{2}{*}{\multicolumn{2}{|c|}{ Species }} & \multicolumn{4}{|c|}{ Location number } & \multirow{3}{*}{$\begin{array}{c}\begin{array}{l}\text { Total No. of } \\
\text { individuals }\end{array} \\
4\end{array}$} \\
\hline & & \multirow{2}{*}{$\begin{array}{l}1 \\
4\end{array}$} & \multirow{2}{*}{2} & \multirow{2}{*}{3} & \multirow{2}{*}{4} & \\
\hline 51. & Agonopterix yeatiana (Fabricius, 1781) & & & & & \\
\hline & Erebidae & & & & & \\
\hline 52. & Amata phegea (Linnaeus, 1758) & 3 & & & & 3 \\
\hline 53. & Arctia villica (Linnaeus, 1758) & & & 3 & & 3 \\
\hline 54. & Arctornis l-nigrum (Muller, 1764) & & & & 1 & 1 \\
\hline 55. & Callimorpha dominula (Linnaeus, 1758) & 4 & & & & 4 \\
\hline 56. & Calliteara pudibunda (Linnaeus, 1758) & & & 5 & 5 & 10 \\
\hline 57. & Catocala fraxini (Linnaeus, 1758) & 4 & & & & 4 \\
\hline 58. & Colobochyla salicalis ([Denis \& Schiffermüller], 1775) & & & 6 & & 6 \\
\hline 59. & Dysauxes ancilla (Linnaeus, 1767) & 1 & & & & 1 \\
\hline 60. & Eilema caniola (Hübner, 1808) & & & & 1 & 1 \\
\hline 61. & Eilema complana (Linnaeus, 1758) & & 6 & & & 6 \\
\hline 62. & Eilema depressa (Esper, 1787) & 4 & 6 & & 1 & 11 \\
\hline 63. & Eilema lurideola (Zincken, 1817) & 2 & & & 1 & 3 \\
\hline 64. & Eilema sororcula (Hufnagel, 1766) & 5 & 12 & 6 & 1 & 24 \\
\hline 65. & Euclidia (Euclidia) glyphica (Linnaeus, 1758) & 1 & & & & 1 \\
\hline 66. & Euplagia quadripunctaria (Poda, 1761) & & & & 1 & 1 \\
\hline 67. & Euproctis (Sphrageidus) similis (Fuessly, 1775) & & & 3 & 1 & 4 \\
\hline 68. & Herminia grisealis ([Denis \& Schiffermüller], 1775) & 2 & 10 & 4 & 2 & 18 \\
\hline 69. & Herminia tarsicrinalis (Knoch, 1782) & 5 & & 7 & 3 & 15 \\
\hline 70. & Herminia tenuialis (Rebel, 1899) & & & 1 & & 1 \\
\hline 71. & Hypena proboscidalis (Linnaeus, 1758) & 2 & & 2 & 2 & 6 \\
\hline 72. & Hypena rostralis (Linnaeus, 1758) & 5 & & & 1 & 6 \\
\hline 73. & Laspeyria flexula ([Denis \& Schiffermüller], 1775) & & & 4 & 1 & 5 \\
\hline 74. & Lithosia quadra (Linnaeus, 1758) & 4 & & 5 & 1 & 10 \\
\hline 75. & Lygephila craccae ([Denis \& Schiffermüller], 1775) & 5 & & & & 5 \\
\hline 76. & Lygephila pastinum (Treitschke, 1826) & & & 1 & & 1 \\
\hline 77. & Lymantria dispar (Linnaeus, 1758) & 4 & 3 & 4 & & 11 \\
\hline 78. & Lymantria monacha (Linnaeus, 1758) & 1 & & 4 & & 5 \\
\hline 79. & Miltochrista miniata (Forster, 1771) & 1 & & 1 & & 2 \\
\hline 80. & Minucia lunaris ([Denis \& Schiffermüller], 1775) & & & & 1 & 1 \\
\hline 81. & Orgyia (Orgyia) antiqua (Linnaeus, 1758) & 1 & 1 & 3 & & 5 \\
\hline 82. & Pechipogo strigilata (Linnaeus, 1758) & 3 & & 3 & & 6 \\
\hline 83. & Pelosia muscerda (Hufnagel, 1766) & & 3 & & & 3 \\
\hline 84. & Penthophera morio (Linnaeus, 1767) & 5 & & & & 5 \\
\hline 85. & Phragmatobia fuliginosa (Linnaeus, 1758) & 2 & 3 & 4 & 1 & 10 \\
\hline 86. & Phytometra viridaria (Clerck, 1759) & 4 & & & & 4 \\
\hline 87. & Polyphaenis sericata (Esper, 1787) & 2 & & 5 & & 7 \\
\hline 88. & Polypogon gryphalis (Herrich-Schäffer, 1851) & 2 & 1 & 1 & & 4 \\
\hline 89. & Polypogon tentacularia (Linnaeus, 1758) & & & 3 & 5 & 8 \\
\hline 90. & Rivula sericealis (Scopoli, 1763) & 2 & 5 & 5 & 5 & 17 \\
\hline 91. & Scoliopteryx libatrix (Linnaeus, 1758) & 5 & & & & 5 \\
\hline 92. & Spilosoma lubricipeda (Linnaeus, 1758) & 7 & 1 & 7 & 6 & 21 \\
\hline 93. & Spilosoma lutea (Hufnagel, 1766) & 4 & & 4 & 7 & 15 \\
\hline 94. & Thumatha senex (Hübner, 1808) & & 2 & & & 2 \\
\hline 95. & Trisateles emortualis ([Denis \& Schiffermüller], 1775) & & & & 2 & 2 \\
\hline
\end{tabular}




\begin{tabular}{|c|c|c|c|c|c|c|}
\hline \multirow{2}{*}{\multicolumn{2}{|c|}{ Species }} & \multicolumn{4}{|c|}{ Location number } & \multirow{2}{*}{$\begin{array}{l}\text { Total No. of } \\
\text { individuals }\end{array}$} \\
\hline & & 1 & 2 & 3 & 4 & \\
\hline & \multicolumn{6}{|l|}{ Geometridae } \\
\hline 96. & Abraxas (Calospilos) sylvata (Scopoli, 1763) & 3 & & & & 3 \\
\hline 97. & Acasis viretata (Hübner, 1799) & 2 & & & 2 & 4 \\
\hline 98. & Aethalura punctulata ([Denis \& Schiffermüller], 1775) & 3 & 21 & & & 24 \\
\hline 99. & Alcis repandata (Linnaeus, 1758) & 5 & & 3 & & 8 \\
\hline 100. & Angerona prunaria (Linnaeus, 1758) & 4 & 2 & & 1 & 7 \\
\hline 101. & Ascotis selenaria ([Denis \& Schiffermüller], 1775) & 1 & & 2 & 1 & 4 \\
\hline 102. & Asthena albulata (Hufnagel, 1767) & 1 & & 9 & & 10 \\
\hline 103. & Asthena anseraria (Herrich-Schäffer, 1855) & & & & 2 & 2 \\
\hline 104. & Biston betularia (Linnaeus, 1758) & 4 & 1 & & & 5 \\
\hline 105. & Cabera exanthemata (Scopoli, 1763) & 5 & 2 & 2 & 1 & 10 \\
\hline 106. & Cabera pusaria (Linnaeus, 1758) & 5 & 1 & & 2 & 8 \\
\hline 107. & Campaea margaritaria (Linnaeus, 1761) & 4 & & 3 & 2 & 9 \\
\hline 108. & Camptogramma bilineata (Linnaeus, 1758) & & & & 1 & 1 \\
\hline 109. & Catarhoe cuculata (Hufnagel, 1767) & 1 & 4 & 2 & 1 & 8 \\
\hline 110. & Chiasmia clathrata (Linnaeus, 1758) & 3 & 1 & 4 & 3 & 11 \\
\hline 111. & Chlorissa cloraria (Hübner, 1813) & & 2 & 4 & & 6 \\
\hline 112. & Chloroclysta siterata (Hufnagel, 1767) & 1 & & & & 1 \\
\hline 113. & Cleora cinctaria ([Denis \& Schiffermüller], 1775) & & 1 & & & 1 \\
\hline 114. & Colostygia pectinataria (Knoch, 1781) & 5 & & & 3 & 8 \\
\hline 115. & Colotois pennaria (Linnaeus, 1761) & & & 4 & & 4 \\
\hline 116. & Cosmorhoe ocellata (Linnaeus, 1758) & & & & 1 & 1 \\
\hline 117. & Cyclophora (Codonia) linearia (Hübner, 1799) & 5 & 3 & 1 & 1 & 10 \\
\hline 118. & Cyclophora (Codonia) porata (Linnaeus, 1767) & & & & 1 & 1 \\
\hline 119. & Cyclophora (Codonia) punctaria (Linnaeus, 1758) & 3 & & 5 & 1 & 9 \\
\hline 120. & Cyclophora (Cyclophora) annularia (Fabricius, 1775) & 8 & 2 & 4 & 6 & 20 \\
\hline 121. & Cyclophora (Cyclophora) pendularia (Clerck, 1759) & & 3 & 2 & & 5 \\
\hline 122. & Cyclophora (Cyclophora) ruficiliaria (Herrich-Schäffer, 1855) & 2 & 1 & & 1 & 4 \\
\hline 123. & Ecliptopera capitata (Herrich-Schäffer, 1839) & & & & 1 & 1 \\
\hline 124. & Ecliptopera silaceata ([Denis \& Schiffermüller], 1775) & 5 & & & & 5 \\
\hline 125. & Ectropis crepuscularia ([Denis \& Schiffermüller], 1775) & 5 & 1 & 2 & 2 & 10 \\
\hline 126. & Ematurga atomaria (Linnaeus, 1758) & 4 & 4 & 5 & 1 & 14 \\
\hline 127. & Ennomos fuscantaria (Haworth, 1809) & & 4 & 4 & & 8 \\
\hline 128. & Ennomos quercinaria (Hufnagel, 1767) & 4 & & & & 4 \\
\hline 129. & Epione repandaria (Hufnagel, 1767) & & & 3 & & 3 \\
\hline 130. & Epirrhoe alternata (Muller, 1764) & 5 & 6 & & 2 & 13 \\
\hline 131. & Erannis defoliaria (Clerck, 1759) & & 2 & & & 2 \\
\hline 132. & Euchoeca nebulata (Scopoli, 1763) & 3 & 4 & & 2 & 9 \\
\hline 133. & Euphyia biangulata (Haworth, 1809) & & & & 1 & 1 \\
\hline 134. & Euphyia unangulata (Haworth, 1809) & 4 & & & & 4 \\
\hline 135. & Gandaritis pyraliata ([Denis \& Schiffermüller], 1775) & & & 2 & & 2 \\
\hline 136. & Geometra papilionaria (Linnaeus, 1758) & 5 & & & & 5 \\
\hline 137. & Gymnoscelis rufifasciata (Haworth, 1809) & & 1 & & 1 & 2 \\
\hline 138. & Heliomata glarearia ([Denis \& Schiffermüller], 1775) & 5 & & & & 5 \\
\hline 139. & Hemistola chrysoprasaria (Esper, 1795) & 4 & & & & 4 \\
\hline 140. & Hemithea aestivaria (Hübner, 1789) & 4 & & 4 & & 8 \\
\hline 141. & Horisme radicaria (de La Harpe, 1855 ) & 5 & & & & 5 \\
\hline
\end{tabular}




\begin{tabular}{|c|c|c|c|c|c|c|}
\hline & & & atio & hum & & Total No. of \\
\hline & species & 1 & 2 & 3 & 4 & individuals \\
\hline 142. & Horisme tersata ([Denis \& Schiffermüller], 1775)* & 1 & & & 3 & 4 \\
\hline 143. & Horisme vitalbata ([Denis \& Schiffermüller], 1775) & 2 & & & & 2 \\
\hline 144. & Hydrelia flammeolaria (Hufnagel, 1767) & 3 & 1 & & & 4 \\
\hline 145. & Hydriomena impluviata ([Denis \& Schiffermüller], 1775) & & & & 5 & 5 \\
\hline 146. & Hypomecis punctinalis (Scopoli, 1763) & 5 & 1 & & 1 & 7 \\
\hline 147. & Hypomecis roboraria ([Denis \& Schiffermüller], 1775) & 5 & 3 & 7 & 3 & 18 \\
\hline 148. & Idaea aversata (Linnaeus, 1758) & 5 & 2 & & 1 & 8 \\
\hline 149. & Idaea degeneraria (Hübner, 1799) & & & & 4 & 4 \\
\hline 150. & Idaea dimidiata (Hufnagel, 1767) & 3 & & & 1 & 4 \\
\hline 151. & Idaea humiliata (Hufnagel, 1767) & 2 & & & & 2 \\
\hline 152. & Idaea muricata (Hufnagel, 1767) & 1 & & & 1 & 2 \\
\hline 153. & Idaea ochrata (Scopoli, 1763) & & 2 & & & 2 \\
\hline 154. & Jodis lactearia (Linnaeus, 1758) & 7 & & 2 & & 9 \\
\hline 155. & Lampropteryx suffumata ([Denis \& Schiffermüller], 1775) & 1 & & & & 1 \\
\hline 156. & Ligdia adustata ([Denis \& Schiffermüller], 1775) & 5 & 3 & 8 & 2 & 18 \\
\hline 157. & Lomaspilis marginata (Linnaeus, 1758) & 6 & & 5 & 2 & 13 \\
\hline 158. & Lomographa bimaculata (Fabricius, 1775) & 2 & 4 & 3 & 1 & 10 \\
\hline 159. & Lomographa temerata ([Denis \& Schiffermüller], 1775) & 5 & 2 & 2 & & 9 \\
\hline 160. & Lycia hirtaria (Clerck, 1759) & & 4 & & & 4 \\
\hline 161. & Macaria alternata ([Denis \& Schiffermüller], 1775) & 6 & 2 & 8 & 6 & 22 \\
\hline 162. & Macaria liturata (Clerck, 1759) & 5 & 1 & 5 & 3 & 14 \\
\hline 163. & Macaria notata (Linnaeus, 1758) & & 1 & 4 & 1 & 6 \\
\hline 164. & Macaria signaria (Hübner, 1809) & 3 & & & & 3 \\
\hline 165. & Melanthia procellata ([Denis \& Schiffermüller], 1775) & 2 & 1 & 5 & 2 & 10 \\
\hline 166. & Mesoleuca albicillata (Linnaeus, 1758) & 10 & 1 & & 4 & 15 \\
\hline 167. & Odontopera bidentata (Clerck, 1759) & 3 & & & & 3 \\
\hline 168. & Operophtera brumata (Linnaeus, 1758)* & & 6 & & & 6 \\
\hline 169. & Ourapteryx sambucaria (Linnaeus, 1758) & & & 3 & & 3 \\
\hline 170. & Parectropis similaria (Hufnagel, 1767) & 4 & 1 & & & 5 \\
\hline 171. & Pareulype berberata ([Denis \& Schiffermüller], 1775) & 5 & & & & 5 \\
\hline 172. & Peribatodes rhomboidaria ([Denis \& Schiffermüller], 1775) & & & 1 & 3 & 4 \\
\hline 173. & Perizoma alchemillata (Linnaeus, 1758) & 5 & 4 & & 1 & 10 \\
\hline 174. & Perizoma flavofasciata (Thunberg, 1792) & 2 & & & & 2 \\
\hline 175. & Perizoma lugdunaria (Herrich-Schäffer, 1855) & 4 & & & & 4 \\
\hline 176. & Petrophora chlorosata (Scopoli, 1763) & 3 & & & & 3 \\
\hline 177. & Phaiogramma etruscaria (Zeller, 1849) & & & 3 & & 3 \\
\hline 178. & Plagodis dolabraria (Linnaeus, 1767) & 1 & 1 & & & 2 \\
\hline 179. & Plagodis pulveraria (Linnaeus, 1758) & 7 & 1 & & & 8 \\
\hline 180. & Pungeleria capreolaria ([Denis \& Schiffermüller], 1775) & 1 & & & & 1 \\
\hline 181. & Scopula (Calothysanis) immutata (Linnaeus, 1758) & & & 5 & 1 & 6 \\
\hline 182. & Scopula (Calothysanis) incanata (Linnaeus, 1758) & & & & 10 & 10 \\
\hline 183. & Scopula (Calothysanis) subpunctaria (Herrich-Schäffer, 1847) & & & 4 & & 4 \\
\hline 184. & Scopula (Scopula) immorata (Linnaeus, 1758) & & 2 & & & 2 \\
\hline 185. & Scopula (Scopula) nigropunctata (Hufnagel, 1767) & & & 6 & 1 & 7 \\
\hline 186. & Scopula (Scopula) ornata (Scopoli, 1763) & 1 & & & & 1 \\
\hline 187. & Scopula (Scopula) tessellaria (Boisduval, 1840) & & & & 2 & 2 \\
\hline 188. & Scotopteryx luridata (Hufnagel, 1767) & 4 & & & & 4 \\
\hline
\end{tabular}




\begin{tabular}{|c|c|c|c|c|c|c|}
\hline \multirow{2}{*}{\multicolumn{2}{|c|}{ Species }} & \multicolumn{4}{|c|}{ Location number } & \multirow{3}{*}{\begin{tabular}{|c}
$\begin{array}{c}\text { Total No. of } \\
\text { individuals }\end{array}$ \\
6 \\
\end{tabular}} \\
\hline & & \multirow{2}{*}{$\begin{array}{l}1 \\
1 \\
\end{array}$} & \multirow{2}{*}{$\begin{array}{l}2 \\
1\end{array}$} & \multirow{2}{*}{$\begin{array}{l}3 \\
4 \\
\end{array}$} & \multirow{2}{*}{4} & \\
\hline 189. & Selenia dentaria (Fabricius, 1775) & & & & & \\
\hline 190. & Selenia lunularia (Hübner, 1788) & 2 & & 1 & 1 & 4 \\
\hline 191. & Selenia tetralunaria (Hufnagel, 1767) & & 5 & 3 & 1 & 9 \\
\hline 192. & Thera variata ([Denis \& Schiffermüller], 1775) & 4 & & & 1 & 5 \\
\hline 193. & Timandra comae Schmidt, 1931 & 1 & & 4 & 3 & 8 \\
\hline 194. & Xanthorhoe designata (Hufnagel, 1767) & 4 & & & & 4 \\
\hline 195. & Xanthorhoe ferrugata (Clerck, 1759) & 6 & 2 & & 11 & 19 \\
\hline 196. & Xanthorhoe fluctuata (Linnaeus, 1758) & 5 & & & & 5 \\
\hline \multirow[t]{2}{*}{197.} & Xanthorhoe spadicearia ([Denis \& Schiffermüller], 1775) & & & 3 & & 3 \\
\hline & Gracillariidae & & & & & \\
\hline \multirow[t]{2}{*}{198.} & Parectopa robiniella Clemens, 1863 & & & & 1 & 1 \\
\hline & Hepialidae & & & & & \\
\hline \multirow[t]{2}{*}{199.} & Triodia sylvina (Linnaeus, 1761) & & & & 1 & 1 \\
\hline & Incurvariidae & & & & & \\
\hline \multirow[t]{2}{*}{200.} & Incurvaria oehlmanniella (Hübner, 1796) & 6 & & & 1 & 7 \\
\hline & Lasiocampidae & & & & & \\
\hline 201. & Euthrix potatoria (Linnaeus, 1758 ) & 3 & & & & 3 \\
\hline 202. & Gastropacha (Gastropacha) quercifolia (Linnaeus, 1758) & 1 & 1 & & & 2 \\
\hline 203. & Lasiocampa (Lasiocampa) quercus (Linnaeus, 1758) & 3 & & & & 3 \\
\hline 204. & Macrothylacia rubi (Linnaeus, 1758) & 5 & & & & 5 \\
\hline 205. & Malacosoma (Clisiocampa) neustria (Linnaeus, 1758) & & & 1 & & 1 \\
\hline 206. & Odonestis pruni (Linnaeus, 1758) & 5 & 1 & & & 6 \\
\hline \multirow[t]{2}{*}{207.} & Phyllodesma (Phyllodesma) tremulifolia (Hübner, 1810) & 5 & & & & 5 \\
\hline & Limacodidae & & & & & \\
\hline 208. & Apoda limacodes (Hufnagel, 1766) & 3 & 1 & 1 & & 5 \\
\hline \multirow[t]{2}{*}{209.} & Heterogenea asella ([Denis \& Schiffermüller], 1775) & & & & 1 & 1 \\
\hline & Noctuidae & & & & & \\
\hline 210. & Acontia (Emmelia) trabealis (Scopoli, 1763) & 2 & 4 & & & 6 \\
\hline 211. & Acronicta (Acronicta) aceris (Linnaeus, 1758) & & 1 & & & 1 \\
\hline 212. & Acronicta (Acronicta) leporina (Linnaeus, 1758) & & 1 & & & 1 \\
\hline 213. & Acronicta (Triaena) cuspis (Hübner, 1813)* & & & & 1 & 1 \\
\hline 214. & Acronicta (Triaena) psi (Linnaeus, 1758) & & & & 11 & 11 \\
\hline 215. & Acronicta (Viminia) euphorbiae ([Denis \& Schiffermüller], 1775) & & & & 1 & 1 \\
\hline 216. & Acronicta (Viminia) rumicis (Linnaeus, 1758) & & 5 & 1 & & 6 \\
\hline 217. & Aedia leucomelas (Linnaeus, 1758) & & 1 & & 1 & 2 \\
\hline 218. & Agrochola (Leptologia) lota (Clerck, 1759) & & & 2 & & 2 \\
\hline 219. & Agrochola (Sunira) circellaris (Hufnagel, 1766) & & 2 & 4 & & 6 \\
\hline 220. & Agrotis exclamationis (Linnaeus, 1758) & & & & 1 & 1 \\
\hline 221. & Agrotis segetum ([Denis \& Schiffermüller], 1775) & & & 5 & & 5 \\
\hline 222. & Allophyes oxyacanthae (Linnaeus, 1758) & & & 4 & 1 & 5 \\
\hline 223. & Amphipyra (Amphipyra) pyramidea (Linnaeus, 1758) & 3 & & & 1 & 4 \\
\hline 224. & Anarta (Calocestra) trifolii (Hufnagel, 1766) & & & & 1 & 1 \\
\hline 225. & Anorthoa munda ([Denis \& Schiffermüller], 1775) & & 2 & & & 2 \\
\hline 226. & Asteroscopus sphinx (Hufnagel, 1766) & & 6 & & 1 & 7 \\
\hline 227. & Autographa gamma (Linnaeus, 1758) & 4 & & 1 & 1 & 6 \\
\hline 228. & Axylia putris (Linnaeus, 1761) & & 4 & 2 & 12 & 18 \\
\hline 229. & Callopistria juventina (Stoll, 1782) & 2 & 2 & 1 & & 5 \\
\hline
\end{tabular}




\begin{tabular}{|c|c|c|c|c|c|c|}
\hline \multirow{2}{*}{\multicolumn{2}{|c|}{ Species }} & \multicolumn{4}{|c|}{ Location number } & \multirow{3}{*}{$\begin{array}{c}\text { Total No. of } \\
\text { individuals }\end{array}$} \\
\hline & & \multirow{2}{*}{1} & \multirow{2}{*}{2} & \multirow{2}{*}{3} & \multirow{2}{*}{$\begin{array}{l}4 \\
1 \\
\end{array}$} & \\
\hline 230. & Caradrina (Paradrina) clavipalpis Scopoli, 1763 & & & & & \\
\hline 231. & Cerastis leucographa ([Denis \& Schiffermüller], 1775) & & 1 & & & 1 \\
\hline 232. & Cerastis rubricosa ([Denis \& Schiffermüller], 1775) & & 3 & & & 3 \\
\hline 233. & Charanyca (Charanyca) trigrammica (Hufnagel, 1766) & & & 2 & & 2 \\
\hline 234. & Charanyca (Rusina) ferruginea (Esper, 1785) & 2 & & & & 2 \\
\hline 235. & Colocasia coryli (Linnaeus, 1758) & 2 & 4 & 4 & 5 & 15 \\
\hline 236. & Conistra (Conistra) ligula (Esper, 1791) & & 1 & & & 1 \\
\hline 237. & Conistra (Conistra) vaccinii (Linnaeus, 1761) & & 1 & 5 & & 6 \\
\hline 238. & Conistra (Dasycampa) erythrocephala ([Denis \& Schiffermüller], 1775) & & 2 & & 1 & 3 \\
\hline 239. & Conistra (Dasycampa) rubiginea ([Denis \& Schiffermüller], 1775) & & 1 & & & 1 \\
\hline 240. & Cosmia (Nemus) pyralina ([Denis \& Schiffermüller], 1775) & 2 & & & & 2 \\
\hline 241. & Craniophora ligustri ([Denis \& Schiffermüller], 1775) & 5 & 3 & & 1 & 9 \\
\hline 242. & Cryphia (Euthales) algae (Fabricius, 1775) & 2 & 3 & & & 5 \\
\hline 243. & Deltote (Deltote) bankiana (Fabricius, 1775) & 4 & 1 & 10 & 2 & 17 \\
\hline 244. & Deltote (Deltote) uncula (Clerck, 1759) & 8 & & & & 8 \\
\hline 245. & Deltote (Protodeltote) pygarga (Hufnagel, 1766) & 2 & 4 & 2 & 6 & 14 \\
\hline 246. & Diachrysia chrysitis (Linnaeus, 1758) & & & & 1 & 1 \\
\hline 247. & Diachrysia stenochrysis (Warren, 1913) & & & & 1 & 1 \\
\hline 248. & Diachrysia zosimi (Hübner, 1822) & & 1 & 1 & & 2 \\
\hline 249. & Diloba caeruleocephala (Linnaeus, 1758) & & & & 1 & 1 \\
\hline 250. & Dypterygia scabriuscula (Linnaeus, 1758) & & & & 2 & 2 \\
\hline 251. & Elaphria venustula (Hübner, 1790) & 5 & & 2 & 11 & 18 \\
\hline 252. & Eucarta amethystina (Hübner, 1803) & 3 & & 3 & 2 & 8 \\
\hline 253. & Eucarta virgo (Treitschke, 1835) & 5 & 5 & 4 & & 14 \\
\hline 254. & Euplexia lucipara (Linnaeus, 1758) & 4 & & & 4 & 8 \\
\hline 255. & Eupsilia transversa (Hufnagel, 1766) & & 2 & 4 & 1 & 7 \\
\hline 256. & Hydraecia micacea (Esper, 1789) & 2 & & & & 2 \\
\hline 257. & Ipimorpha retusa (Linnaeus, 1761) & 1 & 1 & & & 2 \\
\hline 258. & Lacanobia (Dianobia) thalassina (Hufnagel, 1766) & & 1 & & & 1 \\
\hline 259. & Lacanobia (Diataraxia) oleracea (Linnaeus, 1758) & & & 5 & & 5 \\
\hline 260. & Lacanobia (Diataraxia) splendens (Hübner, 1808) & & 1 & & & 1 \\
\hline 261. & Lateroligia ophiogramma (Esper, 1794) & 4 & 1 & 2 & & 7 \\
\hline 262. & Macdunnoughia confusa (Stephens, 1850) & & & & 1 & 1 \\
\hline 263. & Meganephria bimaculosa (Linnaeus, 1767) & & & & 1 & 1 \\
\hline 264. & Melanchra persicariae (Linnaeus, 1761) & 5 & 1 & & & 6 \\
\hline 265. & Mesapamea secalis (Linnaeus, 1758)* & & & & 1 & 1 \\
\hline 266. & Mesoligia furuncula ([Denis \& Schiffermüller], 1775) & 3 & & & & 3 \\
\hline 267. & Moma alpium (Osbeck, 1778) & 1 & 2 & & & 3 \\
\hline 268. & Mormo maura (Linnaeus, 1758) & & & & 1 & 1 \\
\hline 269. & Mythimna (Hyphilare) albipuncta ([Denis \& Schiffermüller], 1775) & & 1 & 4 & & 5 \\
\hline 270. & Mythimna (Hyphilare) congrua (Hübner, 1817) & & 1 & & & 1 \\
\hline 271. & Mythimna (Hyphilare) l-album (Linnaeus, 1767) & & & 3 & & 3 \\
\hline 272. & Mythimna (Mythimna) impura (Hübner, 1808) & 1 & 1 & 1 & & 3 \\
\hline 273. & Mythimna (Mythimna) pallens (Linnaeus, 1758) & & & 2 & 1 & 3 \\
\hline 274. & Mythimna (Mythimna) pudorina ([Denis \& Schiffermüller], 1775) & & & 2 & & 2 \\
\hline 275. & Mythimna (Mythimna) turca (Linnaeus, 1761) & & & 5 & 2 & 7 \\
\hline 276. & Noctua comes Hübner, 1813 & & & 1 & & 1 \\
\hline
\end{tabular}




\begin{tabular}{|c|c|c|c|c|c|c|}
\hline \multirow{2}{*}{\multicolumn{2}{|c|}{ Species }} & \multicolumn{4}{|c|}{ Location number } & \multirow{3}{*}{\begin{tabular}{|c}
$\begin{array}{c}\text { Total No. of } \\
\text { individuals }\end{array}$ \\
5 \\
\end{tabular}} \\
\hline & & \multirow{2}{*}{$\frac{1}{4}$} & \multirow{2}{*}{2} & \multirow{2}{*}{3} & \multirow{2}{*}{$\begin{array}{l}4 \\
1 \\
\end{array}$} & \\
\hline 277. & Noctua fimbriata (Schreber, 1759) & & & & & \\
\hline 278. & Noctua interposita (Hübner, 1790) & & & & 1 & 1 \\
\hline 279. & Noctua orbona (Hufnagel, 1766) & & & 4 & & 4 \\
\hline 280. & Noctua pronuba (Linnaeus, 1758) & & 3 & & 2 & 5 \\
\hline 281. & Ochropleura plecta (Linnaeus, 1761) & 8 & 4 & 8 & 2 & 22 \\
\hline 282. & Oligia latruncula ([Denis \& Schiffermüller], 1775) & 1 & & & & 1 \\
\hline 283. & Oligia strigilis (Linnaeus, 1758) & & & 5 & & 5 \\
\hline 284. & Orthosia (Monima) cerasi (Fabricius, 1775) & & 5 & & & 5 \\
\hline 285. & Orthosia (Monima) cruda ([Denis \& Schiffermüller], 1775) & & 2 & & & 2 \\
\hline 286. & Orthosia (Monima) miniosa ([Denis \& Schiffermüller], 1775) & & 2 & & & 2 \\
\hline 287. & Orthosia (Orthosia) incerta (Hufnagel, 1766) & & 1 & & & 1 \\
\hline 288. & Orthosia (Semiophora) gothica (Linnaeus, 1758) & & 1 & & & 1 \\
\hline 289. & Panolis flammea ([Denis \& Schiffermüller], 1775) & & 1 & & & 1 \\
\hline 290. & Pseudeustrotia candidula ([Denis \& Schiffermüller], 1775) & 1 & 4 & 1 & 21 & 27 \\
\hline 291. & Senta flammea (Curtis, 1828) & & 5 & 2 & & 7 \\
\hline 292. & Sideridis (Aneda) rivularis (Fabricius, 1775 ) & & & & 3 & 3 \\
\hline 293. & Simyra albovenosa (Goeze, 1781) & & 1 & & & 1 \\
\hline 294. & Spodoptera exigua (Hübner, 1808) & & 1 & & & 1 \\
\hline 295. & Tiliacea aurago ([Denis \& Schiffermüller], 1775) & & & 5 & & 5 \\
\hline 296. & Trachea atriplicis (Linnaeus, 1758) & 3 & 1 & 1 & & 5 \\
\hline 297. & Tyta luctuosa ([Denis \& Schiffermüller], 1775) & 4 & 2 & 2 & & 8 \\
\hline 298. & Xanthia (Cirrhia) icteritia (Hufnagel, 1766) & & & 4 & & 4 \\
\hline 299. & Xanthia (Xanthia) togata (Esper, 1788) & & 3 & 4 & 1 & 8 \\
\hline 300. & Xestia (Megasema) c-nigrum (Linnaeus, 1758) & 6 & & 6 & 1 & 13 \\
\hline 301. & Xestia (Megasema) triangulum (Hufnagel, 1766) & & 2 & & & 2 \\
\hline 302. & Xestia (Xestia) castanea (Esper, 1798) & & & & 1 & 1 \\
\hline \multirow[t]{2}{*}{303.} & Xestia (Xestia) stigmatica (Hübner, 1813) & & 2 & & & 2 \\
\hline & Nolidae & & & & & \\
\hline 304. & Bena bicolorana (Fuessly, 1775) & & 2 & & & 2 \\
\hline 305. & Earias clorana (Linnaeus, 1761) & 4 & & 8 & & 12 \\
\hline 306. & Meganola albula ([Denis \& Schiffermüller], 1775) & 4 & & 7 & 1 & 12 \\
\hline 307. & Meganola strigula ([Denis \& Schiffermüller], 1775) & & & & 1 & 1 \\
\hline 308. & Nola chlamitulalis (Hübner, 1813) & 4 & & & & 4 \\
\hline 309. & Nola cristatula (Hübner, 1793) & & 1 & & & 1 \\
\hline 310. & Nycteola asiatica (Krulikovsky, 1904) & & & & 1 & 1 \\
\hline 311. & Nycteola revayana (Scopoli, 1772) & & & & 1 & 1 \\
\hline \multirow[t]{2}{*}{312.} & Pseudoips prasinana (Linnaeus, 1758 ) & 3 & 2 & & 1 & 6 \\
\hline & Notodontidae & & & & & \\
\hline 313. & Clostera anachoreta ([Denis \& Schiffermüller], 1775) & 5 & 7 & & & 12 \\
\hline 314. & Clostera anastomosis (Linnaeus, 1758) & & & & 1 & 1 \\
\hline 315. & Clostera curtula (Linnaeus, 1758) & 1 & & 5 & & 6 \\
\hline 316. & Clostera pigra (Hufnagel, 1766) & 3 & & & 1 & 4 \\
\hline 317. & Drymonia dodonaea ([Denis \& Schiffermüller], 1775) & 1 & & & & 1 \\
\hline 318. & Drymonia melagona (Borkhausen, 1790) & 1 & & 5 & & 6 \\
\hline 319. & Drymonia ruficornis (Hufnagel, 1766) & & 2 & & & 2 \\
\hline 320. & Drymonia velitaris (Hufnagel, 1766) & 2 & & & & 2 \\
\hline 321. & Furcula bicuspis (Borkhausen, 1790) & & & & 1 & 1 \\
\hline
\end{tabular}




\begin{tabular}{|c|c|c|c|c|c|c|}
\hline & & & atio & hum & & Total No. of \\
\hline & Species & 1 & 2 & 3 & 4 & individuals \\
\hline 322. & Furcula furcula (Clerck, 1759) & & 8 & & & 8 \\
\hline 323. & Gluphisia crenata (Esper, 1785) & & & 3 & & 3 \\
\hline 324. & Notodonta dromedarius (Linnaeus, 1767) & & & & 1 & 1 \\
\hline 325. & Notodonta tritophus ([Denis \& Schiffermüller], 1775) & 5 & & & & 5 \\
\hline 326. & Notodonta ziczac (Linnaeus, 1758) & 5 & & & & 5 \\
\hline 327. & Pterostoma palpina (Clerck, 1759) & 5 & & 4 & & 9 \\
\hline 328. & Ptilodon capucina (Linnaeus, 1758) & 10 & & & 1 & 11 \\
\hline 329. & Ptilodon cucullina ([Denis \& Schiffermüller], 1775) & & 1 & 1 & 1 & 3 \\
\hline 330. & Ptilophora plumigera ([Denis \& Schiffermüller], 1775) & & & & 1 & 1 \\
\hline 331. & Spatalia argentina ([Denis \& Schiffermüller], 1775) & 4 & 2 & & 1 & 7 \\
\hline 332. & Stauropus fagi (Linnaeus, 1758) & & 1 & 7 & & 8 \\
\hline & Oecophoridae & & & & & \\
\hline 333. & Harpella forficella (Scopoli, 1763) & 2 & & & & 2 \\
\hline & Peleopodidae & & & & & \\
\hline 334. & Carcina quercana (Fabricius, 1775) & 3 & & 5 & & 8 \\
\hline & Plutellidae & & & & & \\
\hline 335. & Plutella (Plutella) xylostella (Linnaeus, 1758) & & & 5 & 2 & 7 \\
\hline & Psychidae & & & & & \\
\hline 336. & Bijugis bombycella ([Denis \& Schiffermüller], 1775) & & & & 1 & 1 \\
\hline & Pterophoridae & & & & & \\
\hline 337. & Emmelina monodactyla (Linnaeus, 1758) & & & & 1 & 1 \\
\hline 338. & Pterophorus pentadactyla (Linnaeus, 1758) & & 4 & 5 & & 9 \\
\hline & Pyralidae & & & & & \\
\hline 339. & Aphomia sociella (Linnaeus, 1758) & & & 2 & & 2 \\
\hline 340. & Dioryctria abietella ([Denis \& Schiffermüller], 1775) & & 4 & & & 4 \\
\hline 341. & Ematheudes punctella (Treitschke, 1833) & & 4 & & & 4 \\
\hline 342. & Endotricha flammealis ([Denis \& Schiffermüller], 1775) & 3 & & 3 & 1 & 7 \\
\hline 343. & Homoeosoma sinuella (Fabricius, 1794) & & & 4 & & 4 \\
\hline 344. & Hypsopygia costalis (Fabricius, 1775) & 4 & & & & 4 \\
\hline 345. & Nephopterix angustella (Hübner, 1796) & & 4 & & & 4 \\
\hline 346. & Nyctegretis lineana (Scopoli, 1786) & & & & 1 & 1 \\
\hline 347. & Oncocera semirubella (Scopoli, 1763) & 4 & 2 & 4 & 1 & 11 \\
\hline 348. & Phycitodes binaevella (Hübner, 1813) & & 4 & & 1 & 5 \\
\hline 349. & Pyralis regalis Denis \& Schiffermüller, 1775 & & & 4 & & 4 \\
\hline 350. & Sciota adelphella (Fischer v. Röslerstamm, 1836) & 3 & & 4 & 1 & 8 \\
\hline & Saturniidae & & & & & \\
\hline 351. & Aglia tau (Linnaeus, 1758) & & 2 & & & 2 \\
\hline 352. & Antheraea (Antheraea) yamamai (Guérin-Méneville, 1861) & & & & 1 & 1 \\
\hline 353. & Saturnia (Eudia) pavoniella (Scopoli, 1763) & & 1 & & & 1 \\
\hline & Sphingidae & & & & & \\
\hline 354. & Agrius convolvuli (Linnaeus, 1758) & 1 & & & & 1 \\
\hline 355. & Deilephila elpenor (Linnaeus, 1758) & 4 & & 5 & & 9 \\
\hline 356. & Deilephila porcellus (Linnaeus, 1758) & & & 3 & & 3 \\
\hline 357. & Laothoe populi (Linnaeus, 1758) & & & & 1 & 1 \\
\hline 358. & Macroglossum stellatarum (Linnaeus, 1758) & & & & 1 & 1 \\
\hline 359. & Mimas tiliae (Linnaeus, 1758) & 8 & 1 & & & 9 \\
\hline 360. & Smerinthus ocellata (Linnaeus, 1758) & 7 & & & & 7 \\
\hline
\end{tabular}




\begin{tabular}{|c|c|c|c|c|c|c|}
\hline \multirow{2}{*}{\multicolumn{2}{|c|}{ Species }} & \multicolumn{4}{|c|}{ Location number } & \multirow{3}{*}{\begin{tabular}{|c|}
$\begin{array}{l}\text { Total No. of } \\
\text { individuals }\end{array}$ \\
5 \\
\end{tabular}} \\
\hline & & \multirow{2}{*}{1} & \multirow{2}{*}{2} & \multirow{2}{*}{$\frac{3}{5}$} & \multirow{2}{*}{4} & \\
\hline 361. & Sphinx ligustri Linnaeus, 1758 & & & & & \\
\hline \multirow[t]{2}{*}{362.} & Sphinx pinastri Linnaeus, 1758 & 5 & & & 1 & 6 \\
\hline & Tineidae & & & & & \\
\hline \multirow[t]{2}{*}{363.} & Euplocamus anthracinalis (Scopoli, 1763) & & & & 1 & 1 \\
\hline & Tortricidae & & & & & \\
\hline 364. & Acleris hastiana (Linnaeus, 1758$)^{*}$ & & 1 & & & 1 \\
\hline 365. & Agapeta hamana (Linnaeus, 1758) & 1 & 20 & & & 21 \\
\hline 366. & Ancylis mitterbacheriana ([Denis \& Schiffermüller], 1775) & 2 & & & & 2 \\
\hline 367. & Ancylis paludana Barrett, $1871^{*}$ & 4 & & & & 4 \\
\hline 368. & Archips oporana (Linnaeus, 1758) & 5 & & & & 5 \\
\hline 369. & Archips xylosteana (Linnaeus, 1758) & & & & 1 & 1 \\
\hline 370. & Celypha lacunana ([Denis \& Schiffermüller], 1775) & 3 & & & & 3 \\
\hline 371. & Cnephasia (Cnephasia) communana (Herrich-Schäffer, 1851) & 4 & & & & 4 \\
\hline 372. & Epiblema foenella (Linnaeus, 1758) & 5 & 20 & 2 & & 27 \\
\hline 373. & Eulia ministrana (Linnaeus, 1758) & 1 & & & & 1 \\
\hline 374. & Hedya salicella (Linnaeus, 1758) & & 20 & 4 & & 24 \\
\hline 375. & Notocelia cynosbatella (Linnaeus, 1758) & & & 3 & 1 & 4 \\
\hline 376. & Notocelia uddmanniana (Linnaeus, 1758) & & & 5 & & 5 \\
\hline \multirow[t]{2}{*}{377.} & Tortrix viridana Linnaeus, 1758 & 2 & 20 & 1 & & 23 \\
\hline & Yponomeutidae & & & & & \\
\hline \multirow[t]{2}{*}{378.} & Yponomeuta plumbella ([Denis \& Schiffermüller], 1775) & & & & 1 & 1 \\
\hline & Zygaenidae & & & & & \\
\hline 379. & Zygaena (Agrumenia) carniolica (Scopoli, 1763) & & & 5 & & 5 \\
\hline 380. & Zygaena (Mesembrynus) purpuralis (Brünnich, 1763)* & & & 1 & & 1 \\
\hline \multirow[t]{3}{*}{381.} & Zygaena (Zygaena) filipendulae (Linnaeus, 1758) & & & 4 & & 4 \\
\hline & Number of species per locality: & 193 & 149 & 151 & 183 & 381 \\
\hline & Number of specimens per locality: & 693 & 482 & 552 & 353 & 2079 \\
\hline
\end{tabular}

\section{DISCUSSION}

With the 381 moth species recorded during this survey, the area of Bednja River can now be regarded as one of the better surveyed Natura 2000 areas of Croatia in respect to the moth fauna (Vignjević et al., 2011; KorEN \& LADAVAC, 2013; KRANJčEv, 1985). Still, the recorded number probably represents about half of the moth fauna present in the area in comparison with the nearby best surveyed region, Podravina (KRANJČEV, 1985).

In respect to the moth fauna of the county, which now counts 603 species, 189 species were recorded for the first time in the area. The increase is partially because even the existing and mentioned data from the Košćec collection are still unpublished. This unpublished part includes all moth families except Geometridae \& Noctuidae (and partially Erebidae as these species were previously treated within the Noctuidae family) (Kučinić \& Bregović, 1996; Minoci, 2008). Also within Noctuidae and Geometridae a significant increase in the number of species present in the county was evident with this survey. An additional 40 species of Noctuidae and 19 species of Geometridae were recorded as new county records (KučInIĆ \& BREgović, 1996; MiHOci, 2008). It is worth noting that although it is rather short, this is the first moth checklist of any county in Croatia, although hopefully not the last. 


\section{Notes on some interesting species records}

\section{Crambidae}

\section{Catoptria osthelderi (de Lattin, 1950)}

This species is distributed manly in central and northern Europe, with records from France, northern Italy, Romania, Bulgaria and Yugoslavia (SLAmKA, 2012). The adults fly from June till September depending on the location. The larvae feed on various mosses.

Five specimens were collected during this survey. The correct identification of this species was confirmed by the examination of the genital structures (according to SLAMKA, 2012). The only available record that may be traced back to Croatia originates from maps present in Slamka (2012), however, it is not mentioned in the text (except possibly as "Yugoslavia"). No other records from Croatia exist in the literature available to the author (e.g. HABELER, 2008; DE Jong et al., 2014). Due to its presence in the nearby countries of Slovenia and Hungary, additional records are expected in the future across northern Croatia.

\section{Noctuidae}

\section{Diachrysia stenochrysis (Warren, 1913)}

This species is distributed from Europe, through Asia to Japan. It is generally problematic, being part of a cryptic species complex, and certain specimens are hardly distinguishable from one another. In the area of Croatia, the most similar relative is Diachrysia chrysitis (Linnaeus, 1758). The external morphological appearance and the genital structure are very similar in these species. They can be reliably identified only based on the female pheromones (МАсек et al., 2012). One of the better distinguishing features is the shape of the golden area on the fore wing of D. stenochrysis, which is usually well connected (rather H-shaped) while it is regularly discontinuous in $D$. chrysitis. However, the connective golden streak can also be thin or interrupted in $D$. stenochrysis. As the collected specimens show the typical features of $D$. stenochrysis, they were included in the analysis. Further studies of the populations from Croatia needs to be carried out in the future to obtain confirmed data about the occurrence and distribution of this species-pair.

\section{Diachrysia zosimi (Hübner, 1822)}

This is a Trans-Palaearctic marshland species with the "Sibylla-type" distribution pattern, absent or local in Asia Minor. It is widespread but uncommon in Siberia and the Pacific Region, very sporadic in western Asia but present and locally frequent or even common in certain parts of Central and northern Central Europe. In Central Europe it is bivoltine with two more or less continuous generations from the end of May to the end of September. In most parts of its area, the caterpillars feed on both Sanguisorba officinalis and Parnassia palustris L. but preferring Sanguisorba. This species was recorded for the first time in Croatia in the Podravina (KRANJčEV, 1985) and in Turopolje regions (Kučinić \& Perović, 1992/93). The records from the Bednja River contribute to the known distribution of this species in Croatia, and indicate it may be more common than previously thought (MACEK et al., 2008). 


\section{Heterogenea asella ([Denis \& Schiffermüller], 1775)}

This Limacodidae species is usually not very rare, but can be very easily overlooked during surveys, due to its very small size, grey wings and its inconspicuous overall appearance, especially in the cases where only Macroheterocera are studied. This Palaearctic species is distributed in most parts of Europe. Only some scattered records of this species exist from Croatia, most of them from the Podravina region (KRANJčEv, 1985).

\section{CONCLUSIONS}

While the general trend in moth research includes ecological studies on moths (e.g. Highland et al., 2013; Summerville \& Crist, 2003, 2004), Croatia is still without a baseline knowledge of moth fauna. Faunistic surveys would provide basic data for future ecological studies and, if properly done, a baseline for further trend assessments. Such studies are necessary, especially due to the recent changes in land use.

The changes in the human lifestyle within the Varaždin County, and consequently in the land use, have been extreme. For example, in 1890, $91.36 \%$ of the county's inhabitants lived from agriculture (REĐEP \& ŽUGAJ, 1991); this percentage was reduced in 1991 to $13.7 \%$ only, showing a drastic decline (AnOnymous, 2000).

Across the whole Bednja Valley, a clear practice of either complete abandonment or intensification in use of the grasslands is present. Even in the last few years, the changes have been very visible, and the area of wet grasslands is shrinking year by year. With the disappearance of the habitat, plant communities, as well as the connecting fauna (in this case the hygrophilous moths) are also endangered.

The trends in the moth fauna are not known across Europe due to the absence of reference data from previous decades, but for some countries like the Netherlands about one-third of the macromoth species are decreasing (GroenENDIJK \& Ellis, 2011). In order to recognise such trends in Croatia in the future, more baseline surveys are needed.

\section{ACKNOWLEDGMENTS}

I am grateful to Ronkay László for useful corrections of and additions to the manuscript. This moth survey was supported by the Public Institution for the Management of Protected areas of Varaždin County, for which I am eternally grateful.

Received September 22, 2017

\section{REFERENCES}

Anonymous, 2000: Prostorni plan Varaždinske županije, Zavod za prostorno uređenje Varaždinske županije, Varaždin.

ANONYMous, 2015: Natura 2000 Standard Data Forms: HR2001408, HR2001409, HR2001410, HR2001411, HR2001412. Informacijski sustav zaštite prirode, http://www.bioportal.hr/gis/, Hrvatska agencija za okoliš i prirodu, accessed 11. 1. 2017.

GroenendiJK, D. \& ElLis, W.N., 2011: The state of the Dutch larger moth fauna. Journal of Insect Conservation 15, 95-101. 
Grund, A., 1918: Beitrage zur kroatischen Lepidopteren-Fauna. Beitrag C. Lepidopteren der Umgebung von Zagreb (Agram). Glasnik hrvatskog prirodoslovnog društva 30, 59-71.

Fibiger, M., 1997: Noctuidae Europaeae. Vol. 3 (Noctuinae III). Entomological Press, Sorø, 418 pp.

Habeler, H., 2008[2003]: Die Schmetterlinge der Adria-Insel Krk - eine ökofaunistische Studie. Esperiana - Buchreihe zur Entomologie 1-221.

HAHN, M. \& BRÜHL, A. C. 2016: The secret pollinators: an overview of moth pollination with a focus on Europe and North America. Arthropod-Plant Interactions 10(1), 21-28.

Hausmann, A. \& Virdalepp, J, 2012: Geometrid Moths of Europe, 3. Larentiinae 1. Apollo Books, Denmark, 743 pp.

Highland S. A., Miller J.C. \& Jones, J.A., 2013: Determinants of moth diversity and community in a temperate mountain landscape: vegetation, topography, and seasonality. Ecosphere 4(10), 1-22.

de Jong, Y., Verbeek, M., Michelsen, V., Bjørn, P. de P., Los, W., Steeman, F., Bailly, N., Basire, C., Chylarecki, P., Stloukal, E., Hagedorn, G., Wetzel, F., Glöckler, F., Kroupa, A., Korb, G., Hoffmann, A., Häuser, C., Kohlbecker, A., Müller, A., Güntsch, A., Stoev, P. \& Penev, L., 2014: Fauna Europaea - all European animal species on the web. Biodiversity Data Journal 2, 1-35.

JuRINAC, A. E., 1884: Leptiri velikaši (makrolepidoptera) okolice Varaždina. Izvješće Kraljevske velike gimnazije u Varaždinu koncem školske godine 1883/4. Brzotiskom Platzera sina, pp. 55-60.

Kitching, R. L., OrR, A. G., Thalib, L., Mitchell, H., Hopkins, M. S. \& Graham, A. W., 2000: Moth assemblages as indicators of environmental quality in remnants of upland Australian rain forest. Journal of Applied Ecology 37, 284-297.

Koren, T. \& Ladavac, L., 2013: Diversity of Macroheterocera (except fam. Noctuidae [sensu novo] and fam. Geometridae) of central Istria, Croatia. Natura Croatica 22, 73-94.

KRANJČEV, R., 1985: Odnos faune makrolepidoptera prema prirodnim i antropogenim staništima Podravine i podravskih pijesaka (I). Podravski zbornik 11, 200-226.

Kučinić, M. \& Perović, F., 1992: Neke značajke faune sovica (Lepidoptera, Noctuidae) Turopolja (Republika Hrvatska). rad Hrvatske akademije znanosti i umjetnosti. Razred za prirodne znanosti 26, 133149.

Kučinić, M. \& BREgović, A., 1996: A contribution to the knowledge of faunal and zoogeographical characteristics of noctuids (Insecta, Lepidoptera, Noctuidae) in north-western Croatia. Natura Croatica 5, 265-289.

Kučinić, M., Hrašovec, B., Bregović, A. \& Perović, F., 1997. Contribution to the knowledge of clearwing moths (Lepidoptera, Sesiidae) in Croatia. Natura Croatica 6(2), 275-284.

Macek, J., Dvořák, J., Traxler, L. \& Červenka, V., 2008. Noční motýli II. Motýli a housenky střední Evropy - můrovití. Academia, Praha, 490 pp.

MaceK, J., ProcházKa, J. \& Traxler, L., 2012: Noční motýli III. - píd’alkovití. Motýli a housenky střední Evropy. Academia, Praha, 424 pp.

MARČEc, V., 2008: Dnevni leptiri (Insecta, Rhopalocera) zbirke Franje Košćeca Gradskog muzeja Varaždin i komparacija s današnjim stanjem istraživanog područja. Franjo Košćec i njegovo djelo : 1882.-1968. : zbornik radova sa znanstvenog skupa održanog 13. i 14. studenoga 2008. u Varaždinu / [editors M. Šicel, B. Spevec]

Мıносі, I., 2008: Geometrid moths (Lepidoptera, Geometroidea, Geometridae) from the Košćec museum collection. Franjo Košćec i njegovo djelo 1882.-1968. / akademik M. ŠiceL, B. SPEvec (ur.). Zagreb, Varaždin : Hrvatska akademija znanosti i umjetnosti, 129-162.

Nowacki, J., 1998: The noctuids (Lepidoptera, Noctuidae) of Central Europe. František Slamka, Bratislava, $51 \mathrm{pp}$.

PredovniK, Ž., 2010: A revision of clearwing moths (Lepidoptera: Sesiidae) in the collections of the Croatian Natural History Museum in Zagreb and the Entomological Department of the Varaždin Municipal Museum. Natura Croatica 19, 381-388.

REĐEP, M. \& ŽUGAJ, M. 1991: Gospodarske karakteristike Varaždinske županije koncem 19. i početkom 20. stoljeća. Journal of Information and Organizational Sciences 15, 187-202.

Slamka, F., 2006: Pyraloidea of Europe (Lepidoptera) 1. Pyralinae, Galleriinae, Epipaschiinae, Cathariinae \& Odontiinae. František Slamka, Bratislava, 138 pp.

Slamka, F., 2008: Pyraloidea of Europe (Lepidoptera) 2. Crambinae \& Schoenobiinae. František Slamka, Bratislava, 223 pp.

Slamka, F., 2010: Pryaloidea (Lepidoptera) of Central Europe. Identifi cation - Distribution - Habitat Biology. František. Slamka, Bratislava, 112 pp. 
Slamka, F., 2013: Pyraloidea of Europe (Lepidoptera), Volume 3. Pyraustinae \& Spilomelinae. František Slamka, Bratislava: 1-357.

Summerville, K. S. \& T. O. Crist, 2003: Determinants of lepidopteran community composition and species diversity in eastern deciduous forests: roles of season, eco-region and patch size. Oikos 100, 134-148.

Summerville, K. S. \& T. O. Crist, 2004: Contrasting effects of habitat quantity and quality on moth communities in fragmented landscapes. Ecography 27, 3-12.

TавоRSку, V. ,1910: Eine größere Sammeltour im slavischen Süden, Entomologische Zeitschrift, Stuttgart, 23: 224; 230; 24(4), 18-19; 24-25.

Vignjević, G., Zahirović, Ž., Turić, N. \& Merdić, E., 2010: Moths (Lepidoptera: Heterocera) of Kopački rit Nature Park - Results of preliminary research. Entomologia Croatica 14, 17-32.

\section{SAŽETAK}

\section{Raznolikost noćnih leptira (Lepidoptera: Heterocera) u okolici rijeke Bednje, Varaždinska županija, sjeverna Hrvatska}

\section{T. Koren}

Tijekom 2016. godine provedeno je prvo istraživanje noćnih leptira na Natura 2000 lokacijama Livade uz Bednju I - IV i u bližoj okolici. Odabrane su ukupno četiri lokacije, od kojih je svaka bila posjećena po tri puta, u proljeće, ljeto i jesen. Ukupno je zabilježeno 381 vrsta noćnih leptira, a najbrojnije porodice bile su Geometridae (120 vrsta), Noctuidae ( 94 vrste), Erebidae (44 vrste) te Crambidae (35 vrsta). Broj zabilježenih vrsta i jedinki bio je približno isti na svim lokacijama. Kako bismo stavili rezultate u perspektivu, izrađen je prvi preliminarni popis noćnih leptira Varaždinske županije temeljen na dostupnoj literaturi i rezultatima ovog istraživanja koji broji 603 vrste; 189 vrsta po prvi puta je zabilježeno na području županije. Razlog tome je neistraženost te nedostatak objavljenih podataka iz privatnih i muzejskih zbirki. No čak i za porodice koje su istraživane u prošlosti poput sovica i grbica, zabilježeno je povećanje od 40 vrsta (sovice - Noctuidae) te 19 vrsta (Geometridae - grbice) što ukazuje na nedovoljnu istraženost Županije. Daljnjim će se istraživanjima zasigurno udvostručiti broj vrsta poznatih za područje Županije. Neke vrste poput Diachrysia zosimi tek su relativno nedavno zabilježene za faunu Hrvatske i nije poznata njihova točna rasprostranjenost na području Hrvatske. Nakon ovog istraživanja, Natura 2000 područja Livade uz Bednju I - IV mogu se svrstati u najbolje istražene lokacije na području Hrvatske s obzirom na faunu noćnih leptira. 
Appendix I. List of the moths so far recorded in the Varaždin County.

\begin{tabular}{|c|c|c|}
\hline List of species & Literature data & This survey \\
\hline \multicolumn{3}{|l|}{ Alucitidae } \\
\hline Alucita grammodactyla Zeller, 1841 & $\bullet$ & \\
\hline \multicolumn{3}{|l|}{ Bombycidae } \\
\hline Bombyx mori (Linnaeus, 1758) & $\bullet$ & \\
\hline \multicolumn{3}{|l|}{ Chimabachidae } \\
\hline Diurnea fagella ([Denis \& Schiffermüller], 1775) & & $\bullet$ \\
\hline Diurnea lipsiella ([Denis \& Schiffermüller], 1775) & & $\bullet$ \\
\hline \multicolumn{3}{|l|}{ Cossidae } \\
\hline Cossus cossus (Linnaeus, 1758) & $\bullet$ & $\bullet$ \\
\hline Zeuzera pyrina (Linnaeus, 1761) & $\bullet$ & $\bullet$ \\
\hline \multicolumn{3}{|l|}{ Crambidae } \\
\hline Agriphila geniculea (Haworth, 1811) & $\bullet$ & \\
\hline Agriphila tristella ([Denis \& Schiffermüller], 1775) & $\bullet$ & \\
\hline Agrotera nemoralis (Scopoli, 1763) & & $\bullet$ \\
\hline Anania coronata (Hufnagel, 1767) & & $\bullet$ \\
\hline Anania fuscalis ([Denis \& Schiffermüller], 1775) & & $\bullet$ \\
\hline Anania lancealis ([Denis \& Schiffermüller], 1775) & & $\bullet$ \\
\hline Anania stachydalis (Germar, 1821) & & $\bullet$ \\
\hline Anania verbascalis ([Denis \& Schiffermüller], 1775) & & $\bullet$ \\
\hline Calamotropha paludella (Hübner, 1824) & & $\bullet$ \\
\hline Cataclysta lemnata (Linnaeus, 1758) & & - \\
\hline Catoptria osthelderi (Lattin, 1950) & & $\bullet$ \\
\hline Chrysoteuchia culmella (Linnaeus, 1758) & $\bullet$ & $\bullet$ \\
\hline Crambus lathoniellus (Zincken, 1817) & & $\bullet$ \\
\hline Crambus perlella (Scopoli, 1763) & $\bullet$ & \\
\hline Cydalima perspectalis (Walker, 1859) & $\bullet$ & \\
\hline Cynaeda dentalis ([Denis \& Schiffermüller], 1775) & & $\bullet$ \\
\hline Diasemia reticularis (Linnaeus, 1761) & $\bullet$ & $\bullet$ \\
\hline Ecpyrrhorrhoe rubiginalis (Hübner, 1796) & & $\bullet$ \\
\hline Evergestis forficalis (Linnaeus, 1758) & & $\bullet$ \\
\hline Evergestis pallidata (Hufnagel, 1767) & & - \\
\hline Metasia ophialis (Treitschke, 1829) & & $\bullet$ \\
\hline Myelois circumvoluta (Fourcroy, 1785) & & $\bullet$ \\
\hline Nomophila noctuella ([Denis \& Schiffermüller], 1775) & $\bullet$ & $\bullet$ \\
\hline Nymphula nitidulata (Hufnagel, 1767) & & $\bullet$ \\
\hline Ostrinia nubilalis (Hübner, 1796) & & - \\
\hline Palpita vitrealis (Rossi, 1794) & & $\bullet$ \\
\hline Paracorsia repandalis ([Denis \& Schiffermüller], 1775) & & $\bullet$ \\
\hline Parapoynx stratiotata (Linnaeus, 1758) & & $\bullet$ \\
\hline Paratalanta pandalis (Hübner, 1825) & & $\bullet$ \\
\hline Pediasia contaminella (Hübner, 1796) & & $\bullet$ \\
\hline Pleuroptya ruralis (Scopoli, 1763) & & $\bullet$ \\
\hline Pyrausta aurata (Scopoli, 1763) & & $\bullet$ \\
\hline Pyrausta despicata (Scopoli, 1763) & $\bullet$ & $\bullet$ \\
\hline Pyrausta purpuralis (Linnaeus, 1758) & $\bullet$ & $\bullet$ \\
\hline Sclerocona acutella (Eversmann, 1842) & & $\bullet$ \\
\hline Scoparia pyralella ([Denis \& Schiffermüller], 1775) & & - \\
\hline
\end{tabular}




\begin{tabular}{|c|c|c|}
\hline List of species & Literature data & This survey \\
\hline Sitochroa verticalis (Linnaeus, 1758) & & $\bullet$ \\
\hline Udea accolalis (Zeller, 1867) & & - \\
\hline Udea ferrugalis (Hübner, 1796) & & $\bullet$ \\
\hline \multicolumn{3}{|l|}{ Drepanidae } \\
\hline Drepana curvatula (Borkhausen, 1790) & & $\bullet$ \\
\hline Drepana falcataria (Linnaeus, 1758) & & $\bullet$ \\
\hline Falcaria lacertinaria (Linnaeus, 1758) & & $\bullet$ \\
\hline Habrosyne pyritoides (Hufnagel, 1766) & & $\bullet$ \\
\hline Polyploca ridens (Fabricius, 1787 ) & & $\bullet$ \\
\hline Sabra harpagula (Esper, 1786) & & $\bullet$ \\
\hline Tethea or ([Denis \& Schiffermüller], 1775) & & $\bullet$ \\
\hline Thyatira batis (Linnaeus, 1758) & & $\bullet$ \\
\hline Watsonalla binaria (Hufnagel, 1767) & & $\bullet$ \\
\hline Watsonalla cultraria (Fabricius, 1775) & & $\bullet$ \\
\hline \multicolumn{3}{|l|}{ Elachistidae } \\
\hline Agonopterix ocellana (Fabricius, 1775) & & $\bullet$ \\
\hline Agonopterix yeatiana (Fabricius, 1781) & & $\bullet$ \\
\hline \multicolumn{3}{|l|}{ Endromiidae } \\
\hline Endromis versicolora (Linnaeus, 1758) & $\bullet$ & \\
\hline \multicolumn{3}{|l|}{ Erebidae } \\
\hline Amata phegea (Linnaeus, 1758) & $\bullet$ & $\bullet$ \\
\hline Apopestes spectrum (Esper, 1787) & $\bullet$ & \\
\hline Arctia villica (Linnaeus, 1758) & $\bullet$ & $\bullet$ \\
\hline Arctornis l-nigrum (Muller, 1764) & & $\bullet$ \\
\hline Callimorpha dominula (Linnaeus, 1758) & & $\bullet$ \\
\hline Calliteara pudibunda (Linnaeus, 1758) & $\bullet$ & $\bullet$ \\
\hline Catephia alchymista ([Denis \& Schiffermüller], 1775) & $\bullet$ & \\
\hline Catocala electa (Vieweg, 1790) & $\bullet$ & \\
\hline Catocala elocata (Esper, 1787) & - & \\
\hline Catocala fraxini (Linnaeus, 1758) & $\bullet$ & $\bullet$ \\
\hline Catocala fulminea (Scopoli, 1763) & $\bullet$ & \\
\hline Catocala nupta (Linnaeus, 1767) & $\bullet$ & \\
\hline Catocala promissa ([Denis \& Schiffermüller], 1775) & $\bullet$ & \\
\hline Catocala sponsa (Linnaeus, 1767) & $\bullet$ & \\
\hline Colobochyla salicalis ([Denis \& Schiffermüller], 1775) & $\bullet$ & $\bullet$ \\
\hline Cybosia mesomella (Linnaeus, 1758 ) & $\bullet$ & \\
\hline Diacrisia sannio (Linnaeus, 1758) & $\bullet$ & \\
\hline Dicallomera fascelina (Linnaeus, 1758) & $\bullet$ & \\
\hline Dysauxes ancilla (Linnaeus, 1767) & & $\bullet$ \\
\hline Eilema caniola (Hübner, 1808) & & $\bullet$ \\
\hline Eilema complana (Linnaeus, 1758) & & $\bullet$ \\
\hline Eilema depressa (Esper, 1787) & & $\bullet$ \\
\hline Eilema lurideola (Zincken, 1817) & & $\bullet$ \\
\hline Eilema sororcula (Hufnagel, 1766) & & $\bullet$ \\
\hline Euclidia (Euclidia) glyphica (Linnaeus, 1758) & $\bullet$ & $\bullet$ \\
\hline Euplagia quadripunctaria (Poda, 1761) & & $\bullet$ \\
\hline Euproctis (Euproctis) chrysorrhoea (Linnaeus, 1758) & $\bullet$ & \\
\hline Euproctis (Sphrageidus) similis (Fuessly, 1775) & $\bullet$ & $\bullet$ \\
\hline
\end{tabular}




\begin{tabular}{|c|c|c|}
\hline List of species & Literature data & This survey \\
\hline Herminia grisealis ([Denis \& Schiffermüller], 1775) & $\bullet$ & $\bullet$ \\
\hline Herminia tarsicrinalis (Knoch, 1782) & $\bullet$ & $\bullet$ \\
\hline Herminia tarsipennalis (Treitschke, 1835) & $\bullet$ & \\
\hline Herminia tenuialis (Rebel, 1899) & & $\bullet$ \\
\hline Hypena proboscidalis (Linnaeus, 1758 ) & $\bullet$ & $\bullet$ \\
\hline Hypena rostralis (Linnaeus, 1758) & $\bullet$ & $\bullet$ \\
\hline Hyphoraia aulica (Linnaeus, 1758) & $\bullet$ & \\
\hline Idia calvaria ([Denis \& Schiffermüller], 1775) & $\bullet$ & \\
\hline Laspeyria flexula ([Denis \& Schiffermüller], 1775) & $\bullet$ & $\bullet$ \\
\hline Leucoma salicis (Linnaeus, 1758) & $\bullet$ & \\
\hline Lithosia quadra (Linnaeus, 1758) & $\bullet$ & $\bullet$ \\
\hline Lygephila craccae ([Denis \& Schiffermüller], 1775) & $\bullet$ & $\bullet$ \\
\hline Lygephila pastinum (Treitschke, 1826) & $\bullet$ & $\bullet$ \\
\hline Lymantria dispar (Linnaeus, 1758) & $\bullet$ & - \\
\hline Lymantria monacha (Linnaeus, 1758) & $\bullet$ & $\bullet$ \\
\hline Miltochrista miniata (Forster, 1771) & & $\bullet$ \\
\hline Minucia lunaris ([Denis \& Schiffermüller], 1775) & $\bullet$ & $\bullet$ \\
\hline Orgyia (Orgyia) antiqua (Linnaeus, 1758) & & $\bullet$ \\
\hline Paracolax tristalis (Fabricius, 1794) & $\bullet$ & \\
\hline Parascotia fuliginaria (Linnaeus, 1761) & $\bullet$ & \\
\hline Pechipogo strigilata (Linnaeus, 1758) & $\bullet$ & $\bullet$ \\
\hline Pelosia muscerda (Hufnagel, 1766) & & $\bullet$ \\
\hline Penthophera morio (Linnaeus, 1767) & & $\bullet$ \\
\hline Phragmatobia fuliginosa (Linnaeus, 1758) & $\bullet$ & $\bullet$ \\
\hline Phytometra viridaria (Clerck, 1759) & $\bullet$ & $\bullet$ \\
\hline Polyphaenis sericata (Esper, 1787) & & $\bullet$ \\
\hline Polypogon gryphalis (Herrich-Schäffer, 1851) & & $\bullet$ \\
\hline Polypogon tentacularia (Linnaeus, 1758) & $\bullet$ & $\bullet$ \\
\hline Rivula sericealis (Scopoli, 1763) & $\bullet$ & $\bullet$ \\
\hline Scoliopteryx libatrix (Linnaeus, 1758) & $\bullet$ & $\bullet$ \\
\hline Spilosoma lubricipeda (Linnaeus, 1758) & $\bullet$ & $\bullet$ \\
\hline Spilosoma lutea (Hufnagel, 1766) & & $\bullet$ \\
\hline Spilosoma urticae (Esper, 1789) & $\bullet$ & \\
\hline Thumatha senex (Hübner, 1808) & & $\bullet$ \\
\hline Trisateles emortualis ([Denis \& Schiffermüller], 1775) & $\bullet$ & $\bullet$ \\
\hline Tyria jacobaeae (Linnaeus, 1758) & $\bullet$ & \\
\hline Zanclognatha lunalis (Scopoli, 1763) & $\bullet$ & \\
\hline \multicolumn{3}{|l|}{ Geometridae } \\
\hline Abraxas (Abraxas) grossulariata (Linnaeus, 1758 ) & $\bullet$ & \\
\hline Abraxas (Calospilos) sylvata (Scopoli, 1763) & $\bullet$ & $\bullet$ \\
\hline Acasis viretata (Hübner, 1799) & & $\bullet$ \\
\hline Aethalura punctulata ([Denis \& Schiffermüller], 1775) & $\bullet$ & $\bullet$ \\
\hline Agriopis aurantiaria (Hübner, 1799) & $\bullet$ & \\
\hline Agriopis bajaria ([Denis \& Schiffermüller], 1775) & $\bullet$ & \\
\hline Agriopis leucophaearia ([Denis \& Schiffermüller], 1775) & $\bullet$ & \\
\hline Agriopis marginaria (Fabricius, 1776) & $\bullet$ & \\
\hline Alcis repandata (Linnaeus, 1758) & $\bullet$ & $\bullet$ \\
\hline Angerona prunaria (Linnaeus, 1758) & $\bullet$ & $\bullet$ \\
\hline
\end{tabular}




\begin{tabular}{|c|c|c|}
\hline List of species & Literature data & This survey \\
\hline Anticollix sparsata (Treitschke, 1828 ) & $\bullet$ & \\
\hline Apeira syringaria (Linnaeus, 1758) & $\bullet$ & \\
\hline Aplocera plagiata (Linnaeus, 1758) & $\bullet$ & \\
\hline Aplocera praeformata (Hübner, 1826) & $\bullet$ & \\
\hline Artiora evonymaria ([Denis \& Schiffermüller], 1775) & $\bullet$ & \\
\hline Ascotis selenaria ([Denis \& Schiffermüller], 1775) & $\bullet$ & $\bullet$ \\
\hline Asthena albulata (Hufnagel, 1767) & $\bullet$ & $\bullet$ \\
\hline Asthena anseraria (Herrich-Schäffer, 1855) & & $\bullet$ \\
\hline Biston betularia (Linnaeus, 1758) & $\bullet$ & $\bullet$ \\
\hline Cabera exanthemata (Scopoli, 1763) & $\bullet$ & $\bullet$ \\
\hline Cabera pusaria (Linnaeus, 1758) & $\bullet$ & $\bullet$ \\
\hline Campaea margaritaria (Linnaeus, 1761) & $\bullet$ & $\bullet$ \\
\hline Camptogramma bilineata (Linnaeus, 1758) & $\bullet$ & $\bullet$ \\
\hline Catarhoe cuculata (Hufnagel, 1767) & $\bullet$ & $\bullet$ \\
\hline Catarhoe rubidata ([Denis \& Schiffermüller], 1775) & $\bullet$ & \\
\hline Cepphis advenaria (Hübner, 1790) & $\bullet$ & \\
\hline Chiasmia clathrata (Linnaeus, 1758) & $\bullet$ & $\bullet$ \\
\hline Chlorissa cloraria (Hübner, 1813) & $\bullet$ & $\bullet$ \\
\hline Chlorissa viridata (Linnaeus, 1758) & $\bullet$ & \\
\hline Chloroclysta siterata (Hufnagel, 1767) & & $\bullet$ \\
\hline Chloroclystis v-ata (Haworth, 1809) & $\bullet$ & \\
\hline Cleora cinctaria ([Denis \& Schiffermüller], 1775) & & $\bullet$ \\
\hline Colostygia olivata ([Denis \& Schiffermüller], 1775) & $\bullet$ & \\
\hline Colostygia pectinataria (Knoch, 1781) & $\bullet$ & $\bullet$ \\
\hline Colotois pennaria (Linnaeus, 1761) & & $\bullet$ \\
\hline Cosmorhoe ocellata (Linnaeus, 1758) & $\bullet$ & $\bullet$ \\
\hline Crocallis elinguaria (Linnaeus, 1758) & $\bullet$ & \\
\hline Crocallis tusciaria (Borkhausen, 1793) & $\bullet$ & \\
\hline Cyclophora (Codonia) linearia (Hübner, 1799) & $\bullet$ & $\bullet$ \\
\hline Cyclophora (Codonia) porata (Linnaeus, 1767) & $\bullet$ & $\bullet$ \\
\hline Cyclophora (Codonia) punctaria (Linnaeus, 1758) & $\bullet$ & $\bullet$ \\
\hline Cyclophora (Cyclophora) albipunctata (Hufnagel, 1767) & $\bullet$ & \\
\hline Cyclophora (Cyclophora) annularia (Fabricius, 1775) & $\bullet$ & $\bullet$ \\
\hline Cyclophora (Cyclophora) pendularia (Clerck, 1759) & & $\bullet$ \\
\hline Cyclophora (Cyclophora) ruficiliaria (Herrich-Schäffer, 1855) & & - \\
\hline Ecliptopera capitata (Herrich-Schäffer, 1839) & & $\bullet$ \\
\hline Ecliptopera silaceata ([Denis \& Schiffermüller], 1775) & $\bullet$ & $\bullet$ \\
\hline Ectropis crepuscularia ([Denis \& Schiffermüller], 1775) & $\bullet$ & $\bullet$ \\
\hline Electrophaes corylata (Thunberg, 1792) & $\bullet$ & \\
\hline Ematurga atomaria (Linnaeus, 1758) & $\bullet$ & $\bullet$ \\
\hline Ennomos autumnaria (Werneburg, 1859) & $\bullet$ & \\
\hline Ennomos erosaria ([Denis \& Schiffermüller], 1775) & $\bullet$ & \\
\hline Ennomos fuscantaria (Haworth, 1809) & $\bullet$ & $\bullet$ \\
\hline Ennomos quercinaria (Hufnagel, 1767) & $\bullet$ & $\bullet$ \\
\hline Epione repandaria (Hufnagel, 1767) & $\bullet$ & $\bullet$ \\
\hline Epirrhoe alternata (Muller, 1764) & $\bullet$ & $\bullet$ \\
\hline Epirrhoe galiata ([Denis \& Schiffermüller], 1775) & $\bullet$ & \\
\hline Epirrhoe hastulata (Hübner, 1790) & $\bullet$ & \\
\hline
\end{tabular}




\begin{tabular}{|c|c|c|}
\hline List of species & Literature data & This survey \\
\hline Epirrhoe rivata (Hübner, 1813) & - & \\
\hline Epirrhoe tristata (Linnaeus, 1758) & $\bullet$ & \\
\hline Epirrita dilutata ([Denis \& Schiffermüller], 1775) & $\bullet$ & \\
\hline Erannis defoliaria (Clerck, 1759) & $\bullet$ & $\bullet$ \\
\hline Euchoeca nebulata (Scopoli, 1763) & & $\bullet$ \\
\hline Euphyia biangulata (Haworth, 1809) & $\bullet$ & $\bullet$ \\
\hline Euphyia unangulata (Haworth, 1809) & $\bullet$ & $\bullet$ \\
\hline Eupithecia absinthiata (Clerck, 1759) & $\bullet$ & \\
\hline Eupithecia centaureata ([Denis \& Schiffermüller], 1775) & $\bullet$ & \\
\hline Eupithecia denotata (Hübner, 1813) & - & \\
\hline Eupithecia distinctaria Herrich-Schäffer, 1848 & $\bullet$ & \\
\hline Eupithecia inturbata (Hübner, 1817) & $\bullet$ & \\
\hline Eupithecia linariata ([Denis \& Schiffermüller], 1775) & $\bullet$ & \\
\hline Eupithecia plumbeolata (Haworth, 1809) & $\bullet$ & \\
\hline Eupithecia pygmaeata (Hübner, 1799) & $\bullet$ & \\
\hline Eupithecia subfuscata (Haworth, 1809) & - & \\
\hline Eupithecia succenturiata (Linnaeus, 1758) & $\bullet$ & \\
\hline Eupithecia vulgata (Haworth, 1809) & $\bullet$ & \\
\hline Gandaritis pyraliata ([Denis \& Schiffermüller], 1775) & $\bullet$ & $\bullet$ \\
\hline Geometra papilionaria (Linnaeus, 1758) & & $\bullet$ \\
\hline Gnophos (Gnophos) furvata ([Denis \& Schiffermüller], 1775) & $\bullet$ & \\
\hline Gymnoscelis rufifasciata (Haworth, 1809) & $\bullet$ & $\bullet$ \\
\hline Heliomata glarearia ([Denis \& Schiffermüller], 1775) & $\bullet$ & $\bullet$ \\
\hline Hemistola chrysoprasaria (Esper, 1795) & $\bullet$ & $\bullet$ \\
\hline Hemithea aestivaria (Hübner, 1789) & - & - \\
\hline Horisme corticata (Treitschke, 1835) & $\bullet$ & \\
\hline Horisme radicaria (de La Harpe, 1855 ) & & $\bullet$ \\
\hline Horisme tersata ([Denis \& Schiffermüller], 1775) & $\bullet$ & $\bullet$ \\
\hline Horisme vitalbata ([Denis \& Schiffermüller], 1775) & $\bullet$ & $\bullet$ \\
\hline Hydrelia flammeolaria (Hufnagel, 1767) & $\bullet$ & $\bullet$ \\
\hline Hydrelia sylvata ([Denis \& Schiffermüller], 1775) & $\bullet$ & \\
\hline Hydria cervinalis (Scopoli, 1763) & $\bullet$ & \\
\hline Hydria undulata (Linnaeus, 1758) & $\bullet$ & \\
\hline Hydriomena impluviata ([Denis \& Schiffermüller], 1775) & $\bullet$ & $\bullet$ \\
\hline Hypomecis punctinalis (Scopoli, 1763) & $\bullet$ & $\bullet$ \\
\hline Hypomecis roboraria ([Denis \& Schiffermüller], 1775) & - & - \\
\hline Idaea aureolaria ([Denis \& Schiffermüller], 1775) & $\bullet$ & \\
\hline Idaea aversata (Linnaeus, 1758) & $\bullet$ & $\bullet$ \\
\hline Idaea biselata (Hufnagel, 1767) & $\bullet$ & \\
\hline Idaea camparia (Herrich-Schäffer, 1852) & $\bullet$ & \\
\hline Idaea degeneraria (Hübner, 1799) & $\bullet$ & $\bullet$ \\
\hline Idaea dilutaria (Hübner, 1799) & $\bullet$ & \\
\hline Idaea dimidiata (Hufnagel, 1767) & $\bullet$ & $\bullet$ \\
\hline Idaea emarginata (Linnaeus, 1758) & $\bullet$ & \\
\hline Idaea humiliata (Hufnagel, 1767) & $\bullet$ & $\bullet$ \\
\hline Idaea inquinata (Scopoli, 1763) & $\bullet$ & \\
\hline Idaea laevigata (Scopoli, 1763) & - & \\
\hline Idaea moniliata ([Denis \& Schiffermüller], 1775) & $\bullet$ & \\
\hline
\end{tabular}




\begin{tabular}{|c|c|c|}
\hline List of species & Literature data & This survey \\
\hline Idaea muricata (Hufnagel, 1767) & $\bullet$ & $\bullet$ \\
\hline Idaea ochrata (Scopoli, 1763) & $\bullet$ & $\bullet$ \\
\hline Idaea rufaria (Hübner, 1799) & $\bullet$ & \\
\hline Idaea serpentata (Hufnagel, 1767) & $\bullet$ & \\
\hline Idaea straminata (Borkhausen, 1794) & $\bullet$ & \\
\hline Isturgia arenacearia ([Denis \& Schiffermüller], 1775) & $\bullet$ & \\
\hline Isturgia murinaria ([Denis \& Schiffermüller], 1775) & $\bullet$ & \\
\hline Jodis lactearia (Linnaeus, 1758) & $\bullet$ & $\bullet$ \\
\hline Lampropteryx suffumata ([Denis \& Schiffermüller], 1775) & & $\bullet$ \\
\hline Ligdia adustata ([Denis \& Schiffermüller], 1775) & $\bullet$ & $\bullet$ \\
\hline Lithostege griseata ([Denis \& Schiffermüller], 1775) & $\bullet$ & \\
\hline Lomaspilis marginata (Linnaeus, 1758) & $\bullet$ & $\bullet$ \\
\hline Lomographa bimaculata (Fabricius, 1775) & $\bullet$ & $\bullet$ \\
\hline Lomographa temerata ([Denis \& Schiffermüller], 1775) & $\bullet$ & $\bullet$ \\
\hline Lycia hirtaria (Clerck, 1759) & • & • \\
\hline Lycia zonaria ([Denis \& Schiffermüller], 1775) & $\bullet$ & \\
\hline Lythria cruentaria (Hufnagel, 1767) & $\bullet$ & \\
\hline Lythria purpuraria (Linnaeus, 1758) & $\bullet$ & \\
\hline Macaria alternata ([Denis \& Schiffermüller], 1775) & $\bullet$ & $\bullet$ \\
\hline \begin{tabular}{|l} 
Macaria liturata (Clerck, 1759) \\
\end{tabular} & $\bullet$ & $\bullet$ \\
\hline Macaria notata (Linnaeus, 1758) & $\bullet$ & $\bullet$ \\
\hline Macaria signaria (Hübner, 1809) & & $\bullet$ \\
\hline Macaria wauaria (Linnaeus, 1758) & $\bullet$ & \\
\hline Melanthia procellata ([Denis \& Schiffermüller], 1775) & $\bullet$ & $\bullet$ \\
\hline Mesoleuca albicillata (Linnaeus, 1758) & $\bullet$ & $\bullet$ \\
\hline Minoa murinata (Scopoli, 1763) & $\bullet$ & \\
\hline Nebula nebulata (Treitschke, 1828) & $\bullet$ & \\
\hline Nycterosea obstipata (Fabricius, 1794) & $\bullet$ & \\
\hline Odontopera bidentata (Clerck, 1759) & & $\bullet$ \\
\hline Operophtera brumata (Linnaeus, 1758) & $\bullet$ & $\bullet$ \\
\hline Operophtera fagata (Scharfenberg, 1805) & $\bullet$ & \\
\hline Opisthograptis luteolata (Linnaeus, 1758) & $\bullet$ & \\
\hline Ourapteryx sambucaria (Linnaeus, 1758) & & $\bullet$ \\
\hline Pachycnemia hippocastanaria (Hübner, 1799) & $\bullet$ & \\
\hline Parectropis similaria (Hufnagel, 1767) & $\bullet$ & $\bullet$ \\
\hline Pareulype berberata ([Denis \& Schiffermüller], 1775) & $\bullet$ & $\bullet$ \\
\hline Pasiphila debiliata (Hübner, 1817) & $\bullet$ & \\
\hline Pasiphila rectangulata (Linnaeus, 1758) & $\bullet$ & \\
\hline Pelurga comitata (Linnaeus, 1758) & $\bullet$ & \\
\hline Peribatodes rhomboidaria ([Denis \& Schiffermüller], 1775) & $\bullet$ & $\bullet$ \\
\hline Peribatodes secundaria ([Denis \& Schiffermüller], 1775) & $\bullet$ & \\
\hline Perizoma alchemillata (Linnaeus, 1758) & $\bullet$ & $\bullet$ \\
\hline Perizoma flavofasciata (Thunberg, 1792) & $\bullet$ & $\bullet$ \\
\hline Perizoma lugdunaria (Herrich-Schäffer, 1855) & & $\bullet$ \\
\hline Perizoma minorata (Treitschke, 1828 ) & $\bullet$ & \\
\hline Petrophora chlorosata (Scopoli, 1763) & $\bullet$ & $\bullet$ \\
\hline Phaiogramma etruscaria (Zeller, 1849) & & $\bullet$ \\
\hline Philereme transversata (Hufnagel, 1767) & $\bullet$ & \\
\hline
\end{tabular}


134 Koren, T.: Diversity of moths (Lepidoptera: Heterocera) in the surroundings of the Bednja river, Varaždin County

\begin{tabular}{|c|c|c|}
\hline List of species & Literature data & This survey \\
\hline Philereme vetulata ([Denis \& Schiffermüller], 1775) & $\bullet$ & \\
\hline Plagodis dolabraria (Linnaeus, 1767) & $\bullet$ & $\bullet$ \\
\hline Plagodis pulveraria (Linnaeus, 1758) & $\bullet$ & $\bullet$ \\
\hline Plemyria rubiginata ([Denis \& Schiffermüller], 1775) & $\bullet$ & \\
\hline Pseudopanthera macularia (Linnaeus, 1758) & $\bullet$ & \\
\hline Pseudoterpna pruinata (Hufnagel, 1767) & $\bullet$ & \\
\hline Pungeleria capreolaria ([Denis \& Schiffermüller], 1775) & $\bullet$ & $\bullet$ \\
\hline Rheumaptera subhastata (Nolcken, 1870) & $\bullet$ & \\
\hline Rhodometra sacraria (Linnaeus, 1767) & $\bullet$ & \\
\hline Rhodostrophia vibicaria (Clerck, 1759) & $\bullet$ & \\
\hline Scopula (Calothysanis) emutaria (Hübner, 1809) & $\bullet$ & \\
\hline Scopula (Calothysanis) immutata (Linnaeus, 1758) & $\bullet$ & $\bullet$ \\
\hline Scopula (Calothysanis) incanata (Linnaeus, 1758) & & $\bullet$ \\
\hline Scopula (Calothysanis) marginepunctata (Goeze, 1781) & $\bullet$ & \\
\hline Scopula (Calothysanis) subpunctaria (Herrich-Schäffer, 1847) & & $\bullet$ \\
\hline Scopula (Scopula) caricaria (Reutti, 1853) & $\bullet$ & \\
\hline Scopula (Scopula) immorata (Linnaeus, 1758) & $\bullet$ & $\bullet$ \\
\hline Scopula (Scopula) nigropunctata (Hufnagel, 1767) & $\bullet$ & $\bullet$ \\
\hline Scopula (Scopula) ornata (Scopoli, 1763) & $\bullet$ & $\bullet$ \\
\hline Scopula (Scopula) rubiginata (Hufnagel, 1767) & $\bullet$ & \\
\hline Scopula (Scopula) tessellaria (Boisduval, 1840) & & $\bullet$ \\
\hline Scopula (Scopula) virgulata ([Denis \& Schiffermüller], 1775) & $\bullet$ & \\
\hline Scotopteryx bipunctaria ([Denis \& Schiffermüller], 1775) & $\bullet$ & \\
\hline Scotopteryx chenopodiata (Linnaeus, 1758) & $\bullet$ & \\
\hline Scotopteryx luridata (Hufnagel, 1767) & $\bullet$ & $\bullet$ \\
\hline Scotopteryx moeniata (Scopoli, 1763) & $\bullet$ & \\
\hline Selenia dentaria (Fabricius, 1775) & $\bullet$ & $\bullet$ \\
\hline Selenia lunularia (Hübner, 1788) & $\bullet$ & $\bullet$ \\
\hline Selenia tetralunaria (Hufnagel, 1767) & $\bullet$ & $\bullet$ \\
\hline Siona lineata (Scopoli, 1763) & $\bullet$ & \\
\hline Stegania cararia (Hübner, 1790) & $\bullet$ & \\
\hline Stegania dilectaria (Hübner, 1790) & $\bullet$ & \\
\hline Thalera fimbrialis (Scopoli, 1763) & $\bullet$ & \\
\hline Thera variata ([Denis \& Schiffermüller], 1775) & $\bullet$ & $\bullet$ \\
\hline Thetidia (Antonechloris) smaragdaria (Fabricius, 1787) & $\bullet$ & \\
\hline Timandra comae Schmidt, 1931 & $\bullet$ & $\bullet$ \\
\hline Trichopteryx polycommata ([Denis \& Schiffermüller], 1775) & $\bullet$ & \\
\hline Triphosa dubitata (Linnaeus, 1758) & $\bullet$ & \\
\hline Xanthorhoe designata (Hufnagel, 1767) & $\bullet$ & $\bullet$ \\
\hline Xanthorhoe ferrugata (Clerck, 1759) & $\bullet$ & $\bullet$ \\
\hline Xanthorhoe fluctuata (Linnaeus, 1758) & $\bullet$ & $\bullet$ \\
\hline Xanthorhoe montanata ([Denis \& Schiffermüller], 1775) & $\bullet$ & \\
\hline Xanthorhoe quadrifasiata (Clerck, 1759) & $\bullet$ & \\
\hline Xanthorhoe spadicearia ([Denis \& Schiffermüller], 1775) & $\bullet$ & $\bullet$ \\
\hline \multicolumn{3}{|l|}{ Gracillariidae } \\
\hline Parectopa robiniella Clemens, 1863 & & $\bullet$ \\
\hline \multicolumn{3}{|l|}{ Hepialidae } \\
\hline Triodia sylvina (Linnaeus, 1761) & & $\bullet$ \\
\hline
\end{tabular}




\begin{tabular}{|c|c|c|}
\hline List of species & Literature data & This survey \\
\hline \multicolumn{3}{|l|}{ Incurvariidae } \\
\hline Incurvaria oehlmanniella (Hübner, 1796) & & $\bullet$ \\
\hline \multicolumn{3}{|l|}{ Lasiocampidae } \\
\hline Eriogaster (Eriogaster) lanestris (Linnaeus, 1758) & $\bullet$ & \\
\hline Euthrix potatoria (Linnaeus, 1758) & & $\bullet$ \\
\hline Gastropacha (Gastropacha) quercifolia (Linnaeus, 1758) & $\bullet$ & $\bullet$ \\
\hline Lasiocampa (Lasiocampa) quercus (Linnaeus, 1758) & $\bullet$ & $\bullet$ \\
\hline Macrothylacia rubi (Linnaeus, 1758) & $\bullet$ & $\bullet$ \\
\hline Malacosoma (Clisiocampa) neustria (Linnaeus, 1758) & $\bullet$ & $\bullet$ \\
\hline Odonestis pruni (Linnaeus, 1758) & $\bullet$ & $\bullet$ \\
\hline Phyllodesma (Phyllodesma) tremulifolia (Hübner, 1810) & & $\bullet$ \\
\hline \multicolumn{3}{|l|}{ Limacodidae } \\
\hline Apoda limacodes (Hufnagel, 1766) & & • \\
\hline Heterogenea asella ([Denis \& Schiffermüller], 1775) & & $\bullet$ \\
\hline \multicolumn{3}{|l|}{ Noctuidae } \\
\hline Abrostola triplasia (Linnaeus, 1758 ) & $\bullet$ & \\
\hline Acontia (Acontia) lucida (Hufnagel, 1766) & $\bullet$ & \\
\hline Acontia (Emmelia) trabealis (Scopoli, 1763) & $\bullet$ & $\bullet$ \\
\hline Acronicta (Acronicta) aceris (Linnaeus, 1758) & $\bullet$ & $\bullet$ \\
\hline Acronicta (Acronicta) leporina (Linnaeus, 1758) & $\bullet$ & $\bullet$ \\
\hline Acronicta (Hyboma) strigosa ([Denis \& Schiffermüller], 1775) & $\bullet$ & \\
\hline Acronicta (Jocheaera) alni (Linnaeus, 1767) & $\bullet$ & \\
\hline Acronicta (Triaena) cuspis (Hübner, 1813) & & $\bullet$ \\
\hline Acronicta (Triaena) psi (Linnaeus, 1758) & & $\bullet$ \\
\hline Acronicta (Viminia) euphorbiae ([Denis \& Schiffermüller], 1775) & & $\bullet$ \\
\hline Acronicta (Viminia) rumicis (Linnaeus, 1758) & $\bullet$ & $\bullet$ \\
\hline Aedia leucomelas (Linnaeus, 1758) & & $\bullet$ \\
\hline Agrochola (Anchoscelis) litura (Linnaeus, 1758) & $\bullet$ & \\
\hline Agrochola (Leptologia) lota (Clerck, 1759) & & $\bullet$ \\
\hline Agrochola (Leptologia) macilenta (Hübner, 1809) & $\bullet$ & \\
\hline Agrochola (Sunira) circellaris (Hufnagel, 1766) & $\bullet$ & $\bullet$ \\
\hline Agrotis bigramma (Esper, 1790) & $\bullet$ & \\
\hline Agrotis exclamationis (Linnaeus, 1758) & $\bullet$ & $\bullet$ \\
\hline Agrotis ipsilon (Hufnagel, 1766) & $\bullet$ & \\
\hline Agrotis segetum ([Denis \& Schiffermüller], 1775) & & $\bullet$ \\
\hline Allophyes oxyacanthae (Linnaeus, 1758) & & $\bullet$ \\
\hline Amphipyra (Amphipyra) livida ([Denis \& Schiffermüller], 1775) & $\bullet$ & \\
\hline Amphipyra (Amphipyra) pyramidea (Linnaeus, 1758) & $\bullet$ & $\bullet$ \\
\hline Amphipyra (Amphipyra) tragopoginis (Clerck, 1759) & $\bullet$ & \\
\hline Anarta (Anarta) myrtilli (Linnaeus, 1761) & $\bullet$ & \\
\hline Anarta (Calocestra) trifolii (Hufnagel, 1766) & $\bullet$ & $\bullet$ \\
\hline Anorthoa munda ([Denis \& Schiffermüller], 1775) & & $\bullet$ \\
\hline Apamea lithoxylaea ([Denis \& Schiffermüller], 1775) & $\bullet$ & \\
\hline Apamea monoglypha (Hufnagel, 1766) & $\bullet$ & \\
\hline Apamea sordens (Hufnagel, 1766) & $\bullet$ & \\
\hline Asteroscopus sphinx (Hufnagel, 1766) & & $\bullet$ \\
\hline Autographa gamma (Linnaeus, 1758) & $\bullet$ & $\bullet$ \\
\hline Axylia putris (Linnaeus, 1761) & $\bullet$ & $\bullet$ \\
\hline
\end{tabular}




\begin{tabular}{|c|c|c|}
\hline List of species & Literature data & This survey \\
\hline Bryophila (Bryoleuca) raptricula ([Denis \& Schiffermüller], 1775) & $\bullet$ & \\
\hline Bryophila (Bryophila) domestica (Hufnagel, 1766) & $\bullet$ & \\
\hline Callopistria juventina (Stoll, 1782) & $\bullet$ & $\bullet$ \\
\hline Calophasia lunula (Hufnagel, 1766) & $\bullet$ & \\
\hline Calophasia platyptera (Esper, 1788) & $\bullet$ & \\
\hline Caradrina (Paradrina) clavipalpis Scopoli, 1763 & & $\bullet$ \\
\hline Caradrina (Platyperigea) kadenii Freyer, 1836 & $\bullet$ & \\
\hline Cerastis leucographa ([Denis \& Schiffermüller], 1775) & & $\bullet$ \\
\hline Cerastis rubricosa ([Denis \& Schiffermüller], 1775) & $\bullet$ & $\bullet$ \\
\hline Charanyca (Charanyca) trigrammica (Hufnagel, 1766) & $\bullet$ & $\bullet$ \\
\hline Charanyca (Rusina) ferruginea (Esper, 1785) & - & • \\
\hline Colocasia coryli (Linnaeus, 1758) & $\bullet$ & • \\
\hline Conistra (Conistra) ligula (Esper, 1791) & & $\bullet$ \\
\hline Conistra (Conistra) vaccinii (Linnaeus, 1761) & $\bullet$ & $\bullet$ \\
\hline Conistra (Dasycampa) erythrocephala ([Denis \& Schiffermüller], 1775) & & $\bullet$ \\
\hline Conistra (Dasycampa) rubiginea ([Denis \& Schiffermüller], 1775) & $\bullet$ & $\bullet$ \\
\hline Cosmia (Calymnia) trapezina (Linnaeus, 1758$)$ & $\bullet$ & \\
\hline Cosmia (Nemus) pyralina ([Denis \& Schiffermüller], 1775) & & $\bullet$ \\
\hline Craniophora ligustri ([Denis \& Schiffermüller], 1775) & $\bullet$ & $\bullet$ \\
\hline Cryphia (Cryphia) fraudatricula (Hübner, 1803) & $\bullet$ & \\
\hline Cryphia (Cryphia) receptricula (Hübner, 1803) & $\bullet$ & \\
\hline Cryphia (Euthales) algae (Fabricius, 1775) & $\bullet$ & $\bullet$ \\
\hline Cucullia (Cucullia) umbratica (Linnaeus, 1758) & $\bullet$ & \\
\hline Cucullia (Shargacucullia) prenanthis Boisduval, 1840 & $\bullet$ & \\
\hline Cucullia (Shargacucullia) verbasci (Linnaeus, 1758) & $\bullet$ & \\
\hline Deltote (Deltote) bankiana (Fabricius, 1775) & $\bullet$ & $\bullet$ \\
\hline Deltote (Deltote) uncula (Clerck, 1759) & $\bullet$ & $\bullet$ \\
\hline Deltote (Protodeltote) pygarga (Hufnagel, 1766) & $\bullet$ & $\bullet$ \\
\hline Diachrysia chrysitis (Linnaeus, 1758) & $\bullet$ & - \\
\hline Diachrysia chryson (Esper, 1789) & $\bullet$ & \\
\hline Diachrysia stenochrysis (Warren, 1913) & & $\bullet$ \\
\hline Diachrysia zosimi (Hübner, 1822) & & $\bullet$ \\
\hline Dichagyris (Albocosta) flammatra ([Denis \& Schiffermüller], 1775) & $\bullet$ & \\
\hline Diloba caeruleocephala (Linnaeus, 1758$)$ & & $\bullet$ \\
\hline Dypterygia scabriuscula (Linnaeus, 1758) & $\bullet$ & $\bullet$ \\
\hline Egira conspicillaris (Linnaeus, 1758) & $\bullet$ & \\
\hline Elaphria venustula (Hübner, 1790) & & $\bullet$ \\
\hline Eucarta amethystina (Hübner, 1803) & $\bullet$ & $\bullet$ \\
\hline Eucarta virgo (Treitschke, 1835) & & $\bullet$ \\
\hline Euplexia lucipara (Linnaeus, 1758) & $\bullet$ & $\bullet$ \\
\hline Eupsilia transversa (Hufnagel, 1766) & $\bullet$ & $\bullet$ \\
\hline Euxoa (Euxoa) tritici (Linnaeus, 1761) & $\bullet$ & \\
\hline Hada plebeja (Linnaeus, 1761) & $\bullet$ & \\
\hline Hadena (Anepia) perplexa ([Denis \& Schiffermüller], 1775) & $\bullet$ & \\
\hline Hadena (Hadena) bicruris (Hufnagel, 1766) & $\bullet$ & \\
\hline Hecatera bicolorata (Hufnagel, 1766) & $\bullet$ & \\
\hline Hecatera dysodea ([Denis \& Schiffermüller], 1775) & $\bullet$ & \\
\hline Heliothis ononis ([Denis \& Schiffermüller], 1775) & $\bullet$ & \\
\hline
\end{tabular}




\begin{tabular}{|c|c|c|}
\hline List of species & Literature data & This survey \\
\hline Heliothis peltigera ([Denis \& Schiffermüller], 1775) & $\bullet$ & \\
\hline Heliothis viriplaca (Hufnagel, 1766) & $\bullet$ & \\
\hline Hoplodrina ambigua ([Denis \& Schiffermüller], 1775) & $\bullet$ & \\
\hline Hoplodrina superstes (Ochsenheimer, 1816) & $\bullet$ & \\
\hline Hydraecia micacea (Esper, 1789) & & $\bullet$ \\
\hline Ipimorpha retusa (Linnaeus, 1761) & $\bullet$ & $\bullet$ \\
\hline Lacanobia (Dianobia) contigua ([Denis \& Schiffermüller], 1775) & $\bullet$ & \\
\hline Lacanobia (Dianobia) suasa ([Denis \& Schiffermüller], 1775) & $\bullet$ & \\
\hline Lacanobia (Dianobia) thalassina (Hufnagel, 1766) & $\bullet$ & $\bullet$ \\
\hline Lacanobia (Diataraxia) oleracea (Linnaeus, 1758) & $\bullet$ & $\bullet$ \\
\hline Lacanobia (Diataraxia) splendens (Hübner, 1808) & & $\bullet$ \\
\hline Lateroligia ophiogramma (Esper, 1794) & & $\bullet$ \\
\hline Leucania (Leucania) comma (Linnaeus, 1761) & $\bullet$ & \\
\hline Leucania (Leucania) obsoleta (Hübner, 1803) & $\bullet$ & \\
\hline Lithophane (Lithophane) ornitopus (Hufnagel, 1766) & $\bullet$ & \\
\hline Luperina testacea ([Denis \& Schiffermüller], 1775) & $\bullet$ & \\
\hline Macdunnoughia confusa (Stephens, 1850) & $\bullet$ & $\bullet$ \\
\hline Mamestra brassicae (Linnaeus, 1758) & $\bullet$ & \\
\hline Meganephria bimaculosa (Linnaeus, 1767) & & $\bullet$ \\
\hline Melanchra persicariae (Linnaeus, 1761) & $\bullet$ & $\bullet$ \\
\hline Mesapamea secalis (Linnaeus, 1758) & $\bullet$ & $\bullet$ \\
\hline Mesoligia furuncula ([Denis \& Schiffermüller], 1775) & $\bullet$ & $\bullet$ \\
\hline Mniotype satura ([Denis \& Schiffermüller], 1775) & $\bullet$ & \\
\hline Moma alpium (Osbeck, 1778) & & $\bullet$ \\
\hline Mormo maura (Linnaeus, 1758) & $\bullet$ & $\bullet$ \\
\hline Mythimna (Hyphilare) albipuncta ([Denis \& Schiffermüller], 1775) & $\bullet$ & $\bullet$ \\
\hline Mythimna (Hyphilare) congrua (Hübner, 1817) & & $\bullet$ \\
\hline Mythimna (Hyphilare) l-album (Linnaeus, 1767) & $\bullet$ & $\bullet$ \\
\hline Mythimna (Mythimna) impura (Hübner, 1808) & & $\bullet$ \\
\hline Mythimna (Mythimna) pallens (Linnaeus, 1758) & $\bullet$ & $\bullet$ \\
\hline Mythimna (Mythimna) pudorina ([Denis \& Schiffermüller], 1775) & & $\bullet$ \\
\hline Mythimna (Mythimna) turca (Linnaeus, 1761) & $\bullet$ & • \\
\hline Mythimna (Mythimna) vitellina (Hübner, 1808) & $\bullet$ & \\
\hline Naenia typica (Linnaeus, 1758) & $\bullet$ & \\
\hline Noctua comes Hübner, 1813 & & $\bullet$ \\
\hline Noctua fimbriata (Schreber, 1759) & $\bullet$ & $\bullet$ \\
\hline Noctua interposita (Hübner, 1790) & & $\bullet$ \\
\hline Noctua orbona (Hufnagel, 1766) & & $\bullet$ \\
\hline Noctua pronuba (Linnaeus, 1758) & $\bullet$ & $\bullet$ \\
\hline Ochropleura plecta (Linnaeus, 1761) & $\bullet$ & $\bullet$ \\
\hline Oligia latruncula ([Denis \& Schiffermüller], 1775) & $\bullet$ & $\bullet$ \\
\hline Oligia strigilis (Linnaeus, 1758) & $\bullet$ & $\bullet$ \\
\hline Orthosia (Monima) cerasi (Fabricius, 1775 ) & & $\bullet$ \\
\hline Orthosia (Monima) cruda ([Denis \& Schiffermüller], 1775) & & $\bullet$ \\
\hline Orthosia (Monima) miniosa ([Denis \& Schiffermüller], 1775) & & $\bullet$ \\
\hline Orthosia (Orthosia) incerta (Hufnagel, 1766) & $\bullet$ & $\bullet$ \\
\hline Orthosia (Semiophora) gothica (Linnaeus, 1758) & $\bullet$ & $\bullet$ \\
\hline Panemeria tenebrata (Scopoli, 1763) & $\bullet$ & \\
\hline
\end{tabular}




\begin{tabular}{|c|c|c|}
\hline List of species & Literature data & This survey \\
\hline Panolis flammea ([Denis \& Schiffermüller], 1775) & & - \\
\hline Peridroma saucia (Hübner, 1808) & $\bullet$ & \\
\hline Phlogophora meticulosa (Linnaeus, 1758) & $\bullet$ & \\
\hline Plusia festucae (Linnaeus, 1758) & $\bullet$ & \\
\hline Polia nebulosa (Hufnagel, 1766) & $\bullet$ & \\
\hline Pseudeustrotia candidula ([Denis \& Schiffermüller], 1775) & $\bullet$ & $\bullet$ \\
\hline Pyrrhia umbra (Hufnagel, 1766) & $\bullet$ & \\
\hline Rhyacia simulans (Hufnagel, 1766) & $\bullet$ & \\
\hline Senta flammea (Curtis, 1828) & & $\bullet$ \\
\hline Sideridis (Aneda) rivularis (Fabricius, 1775) & - & • \\
\hline Simyra albovenosa (Goeze, 1781) & & $\bullet$ \\
\hline Spaelotis ravida ([Denis \& Schiffermüller], 1775) & $\bullet$ & \\
\hline Spodoptera exigua (Hübner, 1808) & $\bullet$ & $\bullet$ \\
\hline Subacronicta megacephala ([Denis \& Schiffermüller], 1775) & $\bullet$ & \\
\hline Tholera cespitis ([Denis \& Schiffermüller], 1775) & $\bullet$ & \\
\hline Tholera decimalis (Poda, 1761) & $\bullet$ & \\
\hline Tiliacea aurago ([Denis \& Schiffermüller], 1775) & $\bullet$ & $\bullet$ \\
\hline Trachea atriplicis (Linnaeus, 1758) & $\bullet$ & $\bullet$ \\
\hline Tyta luctuosa ([Denis \& Schiffermüller], 1775) & $\bullet$ & $\bullet$ \\
\hline Valeria oleagina ([Denis \& Schiffermüller], 1775) & $\bullet$ & \\
\hline Xanthia (Cirrhia) gilvago ([Denis \& Schiffermüller], 1775) & $\bullet$ & \\
\hline Xanthia (Cirrhia) icteritia (Hufnagel, 1766) & $\bullet$ & $\bullet$ \\
\hline Xanthia (Xanthia) togata (Esper, 1788$)$ & & $\bullet$ \\
\hline Xestia (Megasema) c-nigrum (Linnaeus, 1758) & $\bullet$ & $\bullet$ \\
\hline Xestia (Megasema) ditrapezium ([Denis \& Schiffermüller], 1775) & $\bullet$ & \\
\hline Xestia (Megasema) triangulum (Hufnagel, 1766) & & $\bullet$ \\
\hline Xestia (Xestia) castanea (Esper, 1798) & & $\bullet$ \\
\hline Xestia (Xestia) stigmatica (Hübner, 1813) & & $\bullet$ \\
\hline \multicolumn{3}{|l|}{ Nolidae } \\
\hline Bena bicolorana (Fuessly, 1775) & & $\bullet$ \\
\hline Earias clorana (Linnaeus, 1761) & $\bullet$ & $\bullet$ \\
\hline Earias vernana (Fabricius, 1787 ) & $\bullet$ & \\
\hline Meganola albula ([Denis \& Schiffermüller], 1775) & $\bullet$ & $\bullet$ \\
\hline Meganola strigula ([Denis \& Schiffermüller], 1775) & & $\bullet$ \\
\hline Nola chlamitulalis (Hübner, 1813) & & $\bullet$ \\
\hline Nola cristatula (Hübner, 1793) & & - \\
\hline Nycteola asiatica (Krulikovsky, 1904) & & $\bullet$ \\
\hline Nycteola revayana (Scopoli, 1772) & & $\bullet$ \\
\hline Pseudoips prasinana (Linnaeus, 1758 ) & $\bullet$ & $\bullet$ \\
\hline \multicolumn{3}{|l|}{ Notodontidae } \\
\hline Cerura (Cerura) vinula (Linnaeus, 1758) & $\bullet$ & \\
\hline Clostera anachoreta ([Denis \& Schiffermüller], 1775) & & $\bullet$ \\
\hline Clostera anastomosis (Linnaeus, 1758) & & - \\
\hline Clostera curtula (Linnaeus, 1758) & & $\bullet$ \\
\hline Clostera pigra (Hufnagel, 1766) & & $\bullet$ \\
\hline Drymonia dodonaea ([Denis \& Schiffermüller], 1775) & & $\bullet$ \\
\hline Drymonia melagona (Borkhausen, 1790) & & • \\
\hline Drymonia ruficornis (Hufnagel, 1766) & & $\bullet$ \\
\hline
\end{tabular}




\begin{tabular}{|c|c|c|}
\hline List of species & Literature data & This survey \\
\hline Drymonia velitaris (Hufnagel, 1766) & & $\bullet$ \\
\hline Furcula bicuspis (Borkhausen, 1790) & & $\bullet$ \\
\hline Furcula furcula (Clerck, 1759) & & $\bullet$ \\
\hline Gluphisia crenata (Esper, 1785) & & $\bullet$ \\
\hline Notodonta dromedarius (Linnaeus, 1767) & & $\bullet$ \\
\hline Notodonta tritophus ([Denis \& Schiffermüller], 1775) & & $\bullet$ \\
\hline Notodonta ziczac (Linnaeus, 1758) & & $\bullet$ \\
\hline Phalera bucephala (Linnaeus, 1758) & $\bullet$ & \\
\hline Pterostoma palpina (Clerck, 1759) & & $\bullet$ \\
\hline Ptilodon capucina (Linnaeus, 1758) & & $\bullet$ \\
\hline Ptilodon cucullina ([Denis \& Schiffermüller], 1775) & & $\bullet$ \\
\hline Ptilophora plumigera ([Denis \& Schiffermüller], 1775) & & $\bullet$ \\
\hline Spatalia argentina ([Denis \& Schiffermüller], 1775) & & $\bullet$ \\
\hline Stauropus fagi (Linnaeus, 1758) & & $\bullet$ \\
\hline \multicolumn{3}{|l|}{ Oecophoridae } \\
\hline Harpella forficella (Scopoli, 1763) & & $\bullet$ \\
\hline \multicolumn{3}{|l|}{ Peleopodidae } \\
\hline Carcina quercana (Fabricius, 1775) & & $\bullet$ \\
\hline \multicolumn{3}{|l|}{ Plutellidae } \\
\hline Plutella (Plutella) xylostella (Linnaeus, 1758) & & $\bullet$ \\
\hline \multicolumn{3}{|l|}{ Psychidae } \\
\hline Bijugis bombycella ([Denis \& Schiffermüller], 1775) & & $\bullet$ \\
\hline Canephora hirsuta (Poda, 1761) & $\bullet$ & \\
\hline \multicolumn{3}{|l|}{ Pterophoridae } \\
\hline Emmelina monodactyla (Linnaeus, 1758) & & $\bullet$ \\
\hline Pterophorus pentadactyla (Linnaeus, 1758) & $\bullet$ & $\bullet$ \\
\hline \multicolumn{3}{|l|}{ Pyralidae } \\
\hline Aphomia sociella (Linnaeus, 1758) & & $\bullet$ \\
\hline Dioryctria abietella ([Denis \& Schiffermüller], 1775) & & $\bullet$ \\
\hline Ematheudes punctella (Treitschke, 1833) & & $\bullet$ \\
\hline Endotricha flammealis ([Denis \& Schiffermüller], 1775) & & $\bullet$ \\
\hline Ephestia elutella (Hübner, 1796) & $\bullet$ & \\
\hline Homoeosoma sinuella (Fabricius, 1794) & & $\bullet$ \\
\hline Hypsopygia costalis (Fabricius, 1775) & & $\bullet$ \\
\hline Nephopterix angustella (Hübner, 1796) & & $\bullet$ \\
\hline Nyctegretis lineana (Scopoli, 1786) & & $\bullet$ \\
\hline Oncocera semirubella (Scopoli, 1763) & $\bullet$ & $\bullet$ \\
\hline Phycitodes binaevella (Hübner, 1813) & & $\bullet$ \\
\hline Phycitodes inquinatella (Ragonot, 1887) & $\bullet$ & \\
\hline Plodia interpunctella (Hübner, 1813) & $\bullet$ & \\
\hline Pyralis regalis Denis \& Schiffermüller, 1775 & & $\bullet$ \\
\hline Sciota adelphella (Fischer v. Röslerstamm, 1836) & & $\bullet$ \\
\hline \multicolumn{3}{|l|}{ Saturniidae } \\
\hline Aglia tau (Linnaeus, 1758) & & $\bullet$ \\
\hline Antheraea (Antheraea) yamamai (Guérin-Méneville, 1861) & & $\bullet$ \\
\hline Saturnia (Eudia) pavoniella (Scopoli, 1763) & $\bullet$ & $\bullet$ \\
\hline Saturnia (Saturnia) pyri ([Denis \& Schiffermüller], 1775) & $\bullet$ & \\
\hline
\end{tabular}




\begin{tabular}{|c|c|c|}
\hline List of species & Literature data & This survey \\
\hline \multicolumn{3}{|l|}{ Sesiidae } \\
\hline Bembecia albanensis (Rebel, 1918) & $\bullet$ & \\
\hline Bembecia ichneumoniformis ([Denis \& Schiffermüller], 1775) & $\bullet$ & \\
\hline Bembecia megillaeformis (Hübner, 1813) & $\bullet$ & \\
\hline Chamaesphecia euceraeformis (Ochsenheimer, 1816) & $\bullet$ & \\
\hline Chamaesphecia hungarica (Tomala, 1901) & - & \\
\hline Chamaesphecia nigrifrons (Le Cerf, 1911) & $\bullet$ & \\
\hline Chamaesphecia tenthrediniformis ([Denis \& Schiffermüller], 1775) & $\bullet$ & \\
\hline Paranthrene tabaniformis (Rottemburg, 1775) & $\bullet$ & \\
\hline Pyropteron triannuliformis (Freyer, 1843) & $\bullet$ & \\
\hline Sesia apiformis (Clerck, 1759) & $\bullet$ & \\
\hline Synanthedon cephiformis (Ochsenheimer, 1808) & - & \\
\hline Synanthedon conopiformis (Esper, 1782) & $\bullet$ & \\
\hline Synanthedon culiciformis (Linnaeus, 1758) & $\bullet$ & \\
\hline Synanthedon formicaeformis (Esper, 1783) & $\bullet$ & \\
\hline Synanthedon myopaeformis (Borkhausen, 1789) & $\bullet$ & \\
\hline Synanthedon spheciformis ([Denis \& Schiffermüller], 1775) & $\bullet$ & \\
\hline Synanthedon stomoxiformis (Hübner, 1790) & $\bullet$ & \\
\hline Synanthedon tipuliformis (Clerck, 1759) & $\bullet$ & \\
\hline Synanthedon vespiformis (Linnaeus, 1761) & $\bullet$ & \\
\hline \multicolumn{3}{|l|}{ Sphingidae } \\
\hline Acherontia atropos (Linnaeus, 1758) & $\bullet$ & \\
\hline Agrius convolvuli (Linnaeus, 1758) & $\bullet$ & $\bullet$ \\
\hline Deilephila elpenor (Linnaeus, 1758) & $\bullet$ & $\bullet$ \\
\hline Deilephila porcellus (Linnaeus, 1758) & $\bullet$ & $\bullet$ \\
\hline Hemaris tityus (Linnaeus, 1758) & $\bullet$ & \\
\hline Hyles euphorbiae (Linnaeus, 1758) & $\bullet$ & \\
\hline Laothoe populi (Linnaeus, 1758) & - & - \\
\hline Macroglossum stellatarum (Linnaeus, 1758) & $\bullet$ & $\bullet$ \\
\hline Mimas tiliae (Linnaeus, 1758) & $\bullet$ & $\bullet$ \\
\hline Smerinthus ocellata (Linnaeus, 1758) & $\bullet$ & $\bullet$ \\
\hline Sphinx ligustri Linnaeus, 1758 & $\bullet$ & $\bullet$ \\
\hline Sphinx pinastri Linnaeus, 1758 & & $\bullet$ \\
\hline \multicolumn{3}{|l|}{ Tineidae } \\
\hline Euplocamus anthracinalis (Scopoli, 1763) & & $\bullet$ \\
\hline \multicolumn{3}{|l|}{ Tortricidae } \\
\hline Acleris hastiana (Linnaeus, 1758) & & $\bullet$ \\
\hline Agapeta hamana (Linnaeus, 1758) & & $\bullet$ \\
\hline Ancylis mitterbacheriana ([Denis \& Schiffermüller], 1775) & & - \\
\hline Ancylis paludana Barrett, 1871 & & $\bullet$ \\
\hline Archips oporana (Linnaeus, 1758) & & $\bullet$ \\
\hline Archips xylosteana (Linnaeus, 1758) & & $\bullet$ \\
\hline Celypha lacunana ([Denis \& Schiffermüller], 1775) & & $\bullet$ \\
\hline Celypha rivulana (Scopoli, 1763) & $\bullet$ & \\
\hline Clepsis rurinana (Linnaeus, 1758 ) & $\bullet$ & \\
\hline Cnephasia (Cnephasia) communana (Herrich-Schäffer, 1851) & & $\bullet$ \\
\hline Epiblema foenella (Linnaeus, 1758) & $\bullet$ & $\bullet$ \\
\hline Eulia ministrana (Linnaeus, 1758) & & $\bullet$ \\
\hline
\end{tabular}




\begin{tabular}{|c|c|c|}
\hline List of species & Literature data & This survey \\
\hline Hedya salicella (Linnaeus, 1758) & • & $\bullet$ \\
\hline Notocelia cynosbatella (Linnaeus, 1758) & & $\bullet$ \\
\hline Notocelia uddmanniana (Linnaeus, 1758) & & $\bullet$ \\
\hline Pandemis dumetana (Treitschke, 1835) & - & \\
\hline Tortrix viridana Linnaeus, 1758 & & $\bullet$ \\
\hline \multicolumn{3}{|l|}{ Yponomeutidae } \\
\hline Argyresthia (Argyresthia) bonnetella (Linnaeus, 1758) & - & \\
\hline Yponomeuta plumbella ([Denis \& Schiffermüller], 1775) & & $\bullet$ \\
\hline \multicolumn{3}{|l|}{ Zygaenidae } \\
\hline Zygaena (Agrumenia) carniolica (Scopoli, 1763) & & - \\
\hline Zygaena (Mesembrynus) purpuralis (Brünnich, 1763) & & - \\
\hline Zygaena (Zygaena) filipendulae (Linnaeus, 1758) & - & • \\
\hline Total: & 414 & 381 \\
\hline
\end{tabular}


